\title{
Higher spin de Sitter quasinormal modes
}

\author{
Zimo Sun \\ Department of Physics, Columbia University, \\ 538 West 120th Street, New York, New York 10027, U.S. \\ E-mail: zs2283@columbia.edu
}

ABSTRACT: We construct higher spin quasinormal modes algebraically in $D$-dimensional de Sitter spacetime using the ambient space formalism. The quasinormal modes fall into two nonunitary lowest-weight representations of $\mathfrak{s o}(1, D)$. From a local QFT point of view, the lowest-weight quasinormal modes of massless higher spin fields are produced by gaugeinvariant boundary conserved currents and boundary higher-spin Weyl tensors inserted at the southern pole of the past boundary. We also show that the quasinormal spectrum of a massless/massive spin- $s$ field is precisely encoded in the Harish-Chandra character corresponding to the unitary massless/massive spin- $s \mathrm{SO}(1, D)$ representation.

KeYwords: Higher Spin Gravity, Space-Time Symmetries

ArXiv EPRINT: 2010.09684 


\section{Contents}

1 Introduction 1

2 Ambient space formalism for fields in de Sitter 4

2.1 Higher spin fields in ambient space formalism 5

$\begin{array}{lll}2.2 & \text { Isometry group in ambient space formalism } & 7\end{array}$

3 Algebraic construction of quasinormal modes $\quad 8$

3.1 Scalar fields 8

3.2 Massive higher spin fields $\quad 9$

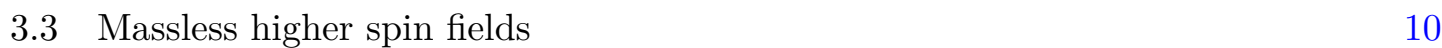

$\begin{array}{lll}3.3 .1 & \text { Maxwell fields } & 12\end{array}$

$\begin{array}{lll}3.3 .2 & \text { Linearized gravity } & 13\end{array}$

$\begin{array}{lll}\text { 3.3.3 Massless higher spin fields } & 15\end{array}$

4 Quasinormal modes from a QFT point of view 17

$\begin{array}{lll}4.1 & \text { Scalar fields } & 17\end{array}$

$\begin{array}{lll}4.2 & \text { Maxwell fields } & 18\end{array}$

5 Quasinormal modes and $\mathrm{SO}(1, d+1)$ characters 20

6 Conclusion and outlook $\quad 24$

A From ambient space to intrinsic coordinate: Maxwell field 26

$\begin{array}{ll}\text { B Match quasinormal spectrums } & 28\end{array}$

C Details of $\gamma_{i_{1} \ell_{1}, \cdots, i_{s} \ell_{s}}^{(s)} \quad 30$

\section{Introduction}

In this paper, we present an algebraic approach to the construction of higher spin quasinormal modes in de Sitter spacetime (dS). In the framework of general relativity (GR), quasinormal modes can be defined as the damped modes of some perturbation in a classical gravitational background with a horizon, like black holes and dS. Astrophysically, they are important because the least damped gravitational quasinormal mode of a Schwarzschild black hole is detected and measured by LIGO through gravitational waves emitted during the so-called "ringdown" phase [1]. The measured value of gravitational quasinormal frequency can be used to test GR which predicts that the spin and mass of a black hole completely fix gravitational quasinormal frequencies. 
One standard method of finding quasinormal modes in a generic background with a horizon is solving the equation of motion for a perturbation, in most cases numerically, and then imposing in-falling boundary condition at the horizon [2-5]. In the static patch of de Sitter spacetime, cf. (3.2), by separation of variables the radial parts of quasinormal modes are found to satisfy hypergeometric functions and hence can be solved analytically (see [6-8] for a summary and derivation of the analytical results associated to scalars, Dirac spinors, Maxwell fields and linearized gravity in any dimensions). The underlying reason for the existence of these analytical solutions is the large dS isometry group which organizes quasinormal modes according to certain representation structure. Such a representation structure was first discovered in $[9,10]$ for a massive scalar field with mass $m^{2}=\Delta(3-$ $\Delta), 0<\Delta<3,{ }^{1}$ in $\mathrm{dS}_{4}$. In this case, the quasinormal modes of the scalar field comprise two (non-unitary) lowest-weight representations of the isometry algebra $\mathfrak{s o}(1,4),{ }^{2}$ which is also the conformal algebra of $\mathbb{R}^{3}$. More explicitly, the authors built two lowest-weight/primary quasinormal modes of quasinormal frequency $i \omega=\Delta$ and $i \omega=\bar{\Delta}=3-\Delta$ respectively, as the two leading asymptotic behaviors of vacuum-to-vacuum bulk two-point function when one point pushed to the northern pole on the future sphere. Upon each primary quasinormal mode, an infinite tower of quasinormal modes can be generated as $\mathfrak{s o}(1,4)$-descendants and the scaling dimension of every descendant can be identified as ( $i \times$ quasinormal frequency). The two towers of quasinormal modes together span the whole scalar quasinormal spectrum. These results were later reformulated by [11] in the ambient space formalism and generalized to massive vector fields and Dirac spinors in the same paper. We will call the way of constructing quasinormal modes using the dS isometry group as in [9-11] the algebraic approach.

The separation of variables method in $[6,7]$ is increasingly cumbersome when applied to higher spin fields due to the rapidly increasing number of tensor structures. So in this paper we will focus on generalizing the algebraic approach to construct quasinormal modes of higher spin fields, which are formulated in the ambient space (see section 2 for a review about the ambient space formalism). In section 3, we first review the algebraic construction of quasinormal modes of a scalar field $\varphi$ of mass $m$ in $\mathrm{dS}_{d+1}$ using the ambient space formalism. The quasinormal spectrum found in this way can be packaged into a "quasinormal character", cf. (5.1)

$$
\chi^{\mathrm{QN}} \equiv \sum_{\omega} d_{\omega} q^{i \omega}
$$

where the sum runs over all quasinormal frequencies and $d_{\omega}$ is the degeneracy of quasinormal modes with frequency $\omega$. We show that the quasinormal character of $\varphi$ is given by

$$
\chi_{\varphi}^{\mathrm{QN}}(q)=\frac{q^{\Delta}+q^{\bar{\Delta}}}{(1-q)^{d}}, \quad \bar{\Delta}=d-\Delta
$$

\footnotetext{
${ }^{1}$ The representation carried by such a scalar field is in the (scalar) complementary series.

${ }^{2}$ Quasinormal modes are defined in the static patch of $\mathrm{dS}_{4}$, which is invariant under the $\mathrm{SO}(1,1) \times \mathrm{SO}(3)$ subgroup of $\mathrm{SO}(1,4)$, though infinitesimally it has the full $\mathfrak{s o}(1,4)$ symmetry. In view of this, the $\mathfrak{s o}(1,4)$ representations carried by quasinormal modes cannot be lifted to an $\mathrm{SO}(1,4)$ action.
} 


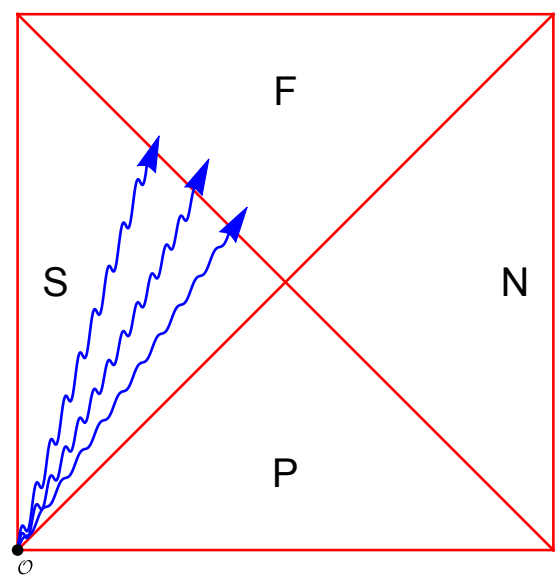

Figure 1: The Penrose diagram of de Sitter spacetime. Quasinormal modes (in the southern static patch "S") of massless higher spin fields are sourced by certain gaugeinvariant operators $\mathcal{O}$ inserted at the southern pole of the past sphere.

where $\Delta=\frac{d}{2}+\sqrt{\frac{d^{2}}{4}-m^{2}}$ is the scaling dimension of $\varphi$. According to [12-14], $\chi_{\varphi}^{\mathrm{QN}}(q)$ is the Harish-Chandra $\mathrm{SO}(1, d+1)$ character corresponding to the unitary (scalar) principal series when $m>\frac{d}{2}$ and the unitary (scalar) complementary series when $0<m \leq \frac{d}{2}$. The $(\Delta \leftrightarrow \bar{\Delta})$ symmetry in (1.2) manifests the two towers of quasinormal modes. The generalization of the algebraic construction to massive higher spin fields is straightforward. The only difference is that the two primary quasinormal modes have spin degeneracy. In this case, the quasinormal character is given by eq. (5.4). However, the generalization to the massless higher spin case is quite nontrivial because gauge symmetry significantly reshapes quasinormal spectrums compared to the massive case. For example, the naive $(\Delta \leftrightarrow \bar{\Delta})$ symmetry would lead to growing modes instead of damped modes because $\bar{\Delta}=2-s<0$ when $s \geq 3$. Moreover the symmetry disagrees with the result of [7] for $s=1,2$. On the representation side, the underlying reason for the difficulty in generalization is that the massless higher spin fields are in the exceptional series for $d \geq 4$ and in the discrete series for $d=3$ while generic massive fields are in the principal series or the complementary series. ${ }^{3}$ In section 3.3, we'll discuss the subtleties associated to gauge symmetry in more detail and explain how to take into account gauge symmetry properly while constructing physical quasinormal modes. In particular, the two-tower structure still holds and the two primary quasinormal modes are given by eq. (3.37) and eq. (3.39). In addition, in section 4 , we argue that the two primary quasinormal modes are produced by insertions of boundary gauge-invariant conserved currents (of scaling dimension $d+s-2$ ) and boundary higher-spin Weyl tensors (of scaling dimension 2) at the southern pole of the past sphere, figure (1). Other quasinormal modes are sourced by the descendants of these two operators.

Based on the algebraic construction described in section 3.3, we extract the physical quasinormal spectrum of massless higher spin fields in section 5 and compute the corresponding quasinormal characters. Here, we list some examples at $d=3,4,5$ (see eq. (5.14)

\footnotetext{
${ }^{3}$ We exclude the partially massless fields while talking about massive fields.
} 
for a general expression working in any $d)$ :

$$
\begin{array}{ll}
d=3: & \chi_{s}^{\mathrm{QN}}(q)=2 \frac{(2 s+1) q^{1+s}-(2 s-1) q^{2+s}}{(1-q)^{3}} \\
d=4: & \chi_{s}^{\mathrm{QN}}(q)=2 \frac{(2 s+1) q^{2}}{(1-q)^{4}} \\
d=5: & \chi_{s}^{\mathrm{QN}}(q)=\frac{1}{3}(s+1)(2 s+1)(2 s+3) \frac{q^{2}-q^{3}}{(1-q)^{5}}+\frac{s+1}{3} \frac{(s+2)(2 s+3) q^{s+3}-s(2 s+1) q^{s+4}}{(1-q)^{5}}
\end{array}
$$

These quasinormal characters coincide with the original computation of Harish-Chandra $\mathrm{SO}(1, d+1)$ characters in $[14,15]$ and the characters appearing in the one-loop path integral of massless higher spin fields on $S^{d+1}$, the Wick rotation of $\mathrm{dS}_{d+1}[16]$. Physically speaking, a Harish-Chandra character can be understood as the trace of $\mathrm{SO}(1, d+1)$ elements over the global single-particle Hilbert space of certain field in $\mathrm{dS}_{d+1}$, which furnishes a unitary irreducible representation of the isometry group $\mathrm{SO}(1, d+1)$. Altogether, the quasinormal characters, which are defined in a pure physics setup, connect nonunitary lowest-weight representations of $\mathfrak{s o}(1, d+1)$ and the unitary representations of $\mathrm{SO}(1, d+1)$. In addition, in the appendix B, we recover the quasinormal spectrums of Maxwell theory and linearized gravity in [7] by using $\chi_{1}^{\mathrm{QN}}(q)$ and $\chi_{2}^{\mathrm{QN}}(q)$.

When $d \geq 4$, the expansion of quasinormal character $\chi_{s}^{\mathrm{QN}}(q)$ always starts from a $q^{2}$ term because it corresponds to a boundary higher-spin Weyl tensor insertion. When $d=3, \chi_{s}^{\mathrm{QN}}(q)$ starts from $q^{1+s}$ since the spin- $s$ Weyl tensor, which vanishes identically on the 3-dimensional boundary, gets replaced by the Cotton tensor, the 3D analogue of Weyl tensor [17-21], which involves $(2 s+1)$ derivatives on the boundary gauge field of scaling dimension $2-s$.

\section{Ambient space formalism for fields in de Sitter}

In this section, we review ambient space formalism for higher spin fields in $(d+1)$ dimensional de Sitter spacetime, which is realized as a hypersurface

$$
\eta_{A B} X^{A} X^{B}=1, \quad \eta_{A B}=(-,+, \cdots,+)
$$

in the ambient space $\mathbb{R}^{1, d+1}$, where $A=0,1, \cdots, d+1$. Local intrinsic coordinates $y^{\mu}$ are defined through an embedding $X^{A}(y)$ that satisfies (2.1) and such an embedding induces a local metric $d s^{2}=g_{\mu \nu} d y^{\mu} d y^{\nu}$ on $\mathrm{dS}_{d+1}$. To gain some intuitions about the ambient space description in field theory, let's consider a free scalar field $\varphi(y)$, defined in the local coordinates $y^{\mu}$, of mass $m^{2}=\Delta(d-\Delta)$ and satisfying equation of motion: $\nabla^{2} \varphi=m^{2} \varphi$ with $\nabla^{2}$ being the scalar Laplacian. This scalar field $\varphi$ admits a unique extension $\phi(X)$ to the ambient space such that

$$
\phi(\lambda X)=\lambda^{-\kappa} \phi(X), \quad \phi(X(y))=\varphi(y)
$$

where $\kappa$ is an arbitrary constant. Define radial coordinate $R=\sqrt{X^{2}}$ and hence points in the ambient space can be parameterized by $\left(R, y^{\mu}\right)$ via a dS foliation. In terms of the radial 
coordinate, the extension condition (2.2) can be rephrased as $\phi(X)=\phi\left(R, y^{\mu}\right)=R^{-\kappa} \varphi(y)$ and the ambient space Laplacian can be expressed as $\partial_{X}^{2}=\frac{1}{R^{d+1}} \partial_{R}\left(R^{d+1} \partial_{R}\right)+\frac{1}{R^{2}} \nabla^{2}$, which together with the equation of motion of $\varphi$, yields

$$
\partial_{X}^{2} \phi=\frac{1}{R^{2}}(\Delta(d-\Delta)-\kappa(d-\kappa)) \phi
$$

In particular, if we choose $\kappa$ to be $\Delta$ or $\bar{\Delta}, \phi(X)$ becomes a harmonic function in the ambient space. Altogether, the scalar field $\varphi(y)$ in $\mathrm{dS}_{d+1}$ of scaling dimension $\Delta$ is equivalent to a homogeneous harmonic function $\phi(X)$ in the ambient space $\mathbb{R}^{1, d+1}$, i.e.

$$
\left(X \cdot \partial_{X}+\kappa\right) \phi(X)=\phi(X), \quad \partial_{X}^{2} \phi(X)=0
$$

where $\kappa=\Delta$ or $\kappa=\bar{\Delta}$. The obvious technical advantage of this ambient space description is replacing the cumbersome covariant derivative $\nabla_{\mu}$ by the simple ordinary derivative $\partial_{X^{A}}$. Such a simplification is more crucial when we deal with higher spin fields. The ambient space formalism for fields of any symmetry type in AdS is described in [22-27], while a detailed discussion specific to the special case of totally symmetric spin- $s$ fields can be found in [28]. For some pedagogical reviews of the totally-symmetric spin- $s$ case under investigation in the current paper see e.g. [29-31]. Here we present an adapted version of the ambient space description in dS.

\subsection{Higher spin fields in ambient space formalism}

The totally symmetric transverse (on-shell) spin- $s$ field $\varphi_{\mu_{1} \ldots \mu_{s}}(y)$ of scaling dimension $\Delta$ in $\mathrm{dS}_{d+1}$ is represented uniquely in ambient space by the symmetric tensor $\phi_{A_{1} \ldots A_{s}}(X)$,

$$
\varphi_{\mu_{1} \cdots \mu_{s}}(y)=\frac{\partial X^{A_{1}}}{\partial y^{\mu_{1}}} \cdots \frac{\partial X^{A_{s}}}{\partial y^{\mu_{s}}} \phi_{A_{1} \cdots A_{s}}(X)
$$

satisfying the following equations:

- Tangentiality to surfaces of constant $R=\sqrt{X^{2}}$ :

$$
\left(X \cdot \partial_{U}\right) \phi_{s}(X, U)=0
$$

- The homogeneity condition:

$$
\left(X \cdot \partial_{X}+\kappa\right) \phi_{s}(X, U)=0
$$

A convenient choice is $\kappa=\Delta$ or $\kappa=\bar{\Delta}$ because, as we have seen in the scalar case, it yields the simplest equation of motion as follows:

- The Casimir condition, i.e. equation of motion

$$
\left(\partial_{X} \cdot \partial_{X}\right) \phi_{s}(X, U)=0
$$

- The transverse condition:

$$
\left(\partial_{X} \cdot \partial_{U}\right) \phi_{s}(X, U)=0
$$


- The traceless condition:

$$
\left(\partial_{U} \cdot \partial_{U}\right) \phi_{s}(X, U)=0
$$

where we have used the generating function $\phi_{s}(X, U) \equiv \frac{1}{s !} \phi_{A_{1} \cdots A_{s}}(X) U^{A_{1}} \cdots U^{A_{s}}$ with $U^{A}$ being a constant auxiliary vector. The first two conditions ensure that $\phi_{s}(X, U)$ is the unique uplift of $\varphi_{\mu_{1} \cdots \mu_{s}}$ that satisfies (2.5) and the last three conditions are equivalent to the Fierz-Pauli system:

The Casimir condition : $\quad \nabla^{2} \varphi_{\mu_{1} \cdots \mu_{s}}=(\Delta(d-\Delta)+s) \varphi_{\mu_{1} \cdots \mu_{s}}$

The transverse condition : $\quad \nabla^{\mu_{1}} \varphi_{\mu_{1} \cdots \mu_{s}}=0$

The traceless condition : $\quad g^{\mu_{1} \mu_{2}} \varphi_{\mu_{1} \cdots \mu_{s}}=0$

In the remaining part of the paper, we will call (2.6)-(2.7) the uplift conditions and (2.8)(2.10) the Fierz-Pauli conditions.

When $\varphi_{\mu_{1} \cdots \mu_{s}}$ is a massless spin- $s$ bulk field, the uplift conditions and Fierz-Pauli conditions have a gauge symmetry, with the gauge transformation takes the following simple form if we choose $\kappa=2-s=\bar{\Delta}$ in eq. (2.7):

$$
\delta_{\xi_{s-1}} \phi_{s}(X, U)=\left(U \cdot \partial_{X}\right) \xi_{s-1}(X, U)
$$

where $\xi_{s-1}$ satisfies

- Tangentiality to surfaces of constant $R=\sqrt{X^{2}}$ :

$$
\left(X \cdot \partial_{U}\right) \xi_{s-1}(X, U)=0
$$

- The homogeneity condition:

$$
\left(X \cdot \partial_{X}+1-s\right) \xi_{s-1}(X, U)=0
$$

- The Casimir condition:

$$
\left(\partial_{X} \cdot \partial_{X}\right) \xi_{s-1}(X, U)=0
$$

- The transverse condition:

$$
\left(\partial_{X} \cdot \partial_{U}\right) \xi_{s-1}(X, U)=0
$$

- The traceless condition:

$$
\left(\partial_{U} \cdot \partial_{U}\right) \xi_{s-1}(X, U)=0
$$

In the intrinsic coordinate language, this set of equations implies that $\xi_{s-1}$ is a transverse traceless symmetric spin- $(s-1)$ field on $\mathrm{dS}_{d+1}$ satisfying on-shell equation of motion $\left(\nabla^{2}-(s-1)(s+d-2)\right) \xi_{s-1}=0$, where spin indices are suppressed. 


\subsection{Isometry group in ambient space formalism}

$\mathrm{dS}_{d+1}$ is a maximally symmetric space with isometry group $\mathrm{SO}(1, d+1)$ generated by $L_{A B}=-L_{B A}$, subject to the commutation relations:

$$
\left[L_{A B}, L_{C D}\right]=\eta_{B C} L_{A D}+\eta_{D B} L_{C A}+\eta_{A D} L_{B C}+\eta_{C A} L_{D B}
$$

For unitary representations, $L_{A B}$ are anti-hermitian. The following linear combinations of $L_{A B}$

$$
D=L_{0, d+1}, \quad M_{i j}=L_{i j}, \quad P_{i}=L_{d+1, i}+L_{0, i}, \quad K_{i}=L_{d+1, i}-L_{0, i}
$$

lead to the conformal algebra of $\mathbb{R}^{d}$. Here we list some nontrivial commutators of the conformal algebra

$$
\begin{aligned}
& {\left[D, P_{i}\right]=P_{i}, \quad\left[D, K_{i}\right]=-K_{i}, \quad\left[K_{i}, P_{j}\right]=2 \delta_{i j} D-2 M_{i j}} \\
& {\left[M_{i j}, P_{k}\right]=\delta_{j k} P_{i}-\delta_{i k} P_{j}, \quad\left[M_{i j}, K_{k}\right]=\delta_{j k} K_{i}-\delta_{i k} K_{j}}
\end{aligned}
$$

$\mathrm{SO}(1, d+1)$ acts linearly on fields in the ambient space $\mathbb{R}^{1, d+1}$. In particular, the generators $L_{A B}$ are realized as linear differential operators in both $X$ and $U$ :

$$
L_{A B}=\left(X_{A} \partial_{X^{B}}-X_{B} \partial_{X^{A}}\right)+\left(U_{A} \partial_{U^{B}}-U_{B} \partial_{U^{A}}\right)
$$

where the first term corresponds to orbital angular momentum and the second term represents spin angular momentum. The action of $M_{i j}, P_{i}, K_{i}, D$ induced by $(2.23)$ is

$$
\begin{aligned}
M_{i j} & =X_{i} \partial_{X^{j}}-X_{j} \partial_{X^{i}}+U_{i} \partial_{U^{j}}-U_{j} \partial_{U^{i}} \\
K_{i} & =X^{+} \partial_{X^{i}}+2 X_{i} \partial_{X^{-}}+U^{+} \partial_{U^{i}}+2 U_{i} \partial_{U^{-}} \\
P_{i} & =-X^{-} \partial_{X^{i}}-2 X_{i} \partial_{X^{+}}-U^{-} \partial_{U^{i}}-2 U_{i} \partial_{U^{+}} \\
D & =-X^{+} \partial_{X^{+}}+X^{-} \partial_{X^{-}}-U^{+} \partial_{U^{+}}+U^{-} \partial_{U^{-}}
\end{aligned}
$$

where we have used lightcone coordinates $X^{ \pm} \equiv X^{0} \pm X^{d+1}$ and $U^{ \pm} \equiv U^{0} \pm U^{d+1}$.

With a little algebra, one can show that all $L_{A B}$ commute with the following set of differential operators:

$$
X \cdot \partial_{U}, \quad X \cdot \partial_{X}, \quad \partial_{X}^{2}, \quad \partial_{X} \cdot \partial_{U}, \quad \partial_{U}^{2}, \quad U \cdot \partial_{X}
$$

The first five operators define the uplift conditions and Fierz-Pauli conditions, cf. (2.6)(2.10), and hence the commutation relations imply that the on-shell bulk fields carry representations of $\mathfrak{s o}(1, d+1)$. The last operator defines the gauge transformation of a massless higher spin field and therefore one implication of $\left[L_{A B}, U \cdot \partial_{X}\right]=0$ is that the descendants of a pure gauge mode are also pure gauge. This observation is crucial when we construct quasinormal modes for massless higher spin fields. 


\section{Algebraic construction of quasinormal modes}

The southern static patch of de Sitter spacetime, which corresponds to the region denoted by "S" in the figure 1 , has coordinates

$$
X^{0}=\sqrt{1-r^{2}} \sinh t, \quad X^{i}=r \Omega^{i}, \quad X^{d+1}=\sqrt{1-r^{2}} \cosh t
$$

and shows a manifest spherical horizon at $r=1$ or $\rho=\infty$ in its metric

$$
\begin{aligned}
d s^{2} & =-\left(1-r^{2}\right) d t^{2}+\frac{d r^{2}}{1-r^{2}}+r^{2} d \Omega^{2} \\
& =\frac{-d t^{2}+d \rho^{2}}{\cosh ^{2} \rho}+\tanh ^{2} \rho d \Omega^{2}
\end{aligned}
$$

where $r=\tanh \rho$ and $d \Omega^{2}=h_{a b} d \vartheta^{a} d \vartheta^{b}$ is the standard metric on $S^{d-1}$ ( $\vartheta^{a}$ are spherical coordinates on $S^{d-1}$ ). The traditional analytical approach to quasinormal requires solving the equation of motion in bulk and imposing in-falling boundary condition, i.e. $e^{-i \omega_{\mathrm{QN}}(t-\rho)}$, for the leading asymptotic behavior near the horizon at $\rho=\infty$.

An algebraic method of solving quasinormal modes was first used for scalar fields in $\mathrm{dS}_{4}$ in $[9,10]$. In particular, the authors found that all the quasinormal modes fall into two lowest-weight representations of the conformal algebra $\mathfrak{s o}(1, d+1)$. Therefore, it suffices to find the two lowest-weight/primary quasinormal modes, which are solutions to the equation of motion and are annihilated by $K_{i}$, and the rest of the quasinormal spectrum can be generated as descendants of them. In this section, we will first reformulate this scalar story using the ambient space formalism and then generalize it to higher spin fields.

\subsection{Scalar fields}

Let $\varphi(X)$ be a free scalar field of mass $m^{2}=\Delta(d-\Delta)>0$ in $\mathrm{dS}_{d+1}$. By construction, the equation of motion $\left(\nabla^{2}-m^{2}\right) \varphi=0$ is satisfied by the boundary-to-bulk propagators, which in ambient space take the following form:

$$
\alpha_{\Delta}(X ; \xi)=\frac{1}{(X \cdot \xi)^{\Delta}}, \quad \beta_{\Delta}(X ; \xi)=\frac{1}{(X \cdot \xi)^{\bar{\Delta}}}
$$

where $\xi^{A}$ is a constant null vector in $\mathbb{R}^{1, d+1}$ representing a point on the future/past boundary of $\mathrm{dS}_{d+1}$. Treating $\alpha_{\Delta}$ and $\beta_{\Delta}$ as mode functions in $X^{A}$, they are primary with respect to the conformal algebra $\mathfrak{s o}(1, d+1)$ if $X \cdot \xi=X^{+}$, because $K_{i}$ only involves derivatives $\partial_{X^{i}}$ and $\partial_{X^{-}}$while acting on scalar fields (cf. (2.24)). By choosing $\xi^{A}=(-1,0, \cdots, 0,1)$ which is the southern pole of the past sphere, we obtain the two primary quasinormal modes

$$
\begin{aligned}
& \alpha_{\Delta}(X)=\frac{1}{\left(X^{+}\right)^{\Delta}}=(\cosh \rho)^{\Delta} e^{-\Delta t \rho \underset{\sim}{\sim \rightarrow \infty} e^{-\Delta(t-\rho)}} \\
& \beta_{\Delta}(X)=\frac{1}{\left(X^{+}\right)^{\bar{\Delta}}}=(\cosh \rho)^{\bar{\Delta}} e^{-\bar{\Delta} t \rho \rightarrow \infty} e^{-\bar{\Delta}(t-\rho)}
\end{aligned}
$$

with quasinormal frequency $i \omega_{\alpha}=\Delta$ and $i \omega_{\beta}=\bar{\Delta}$ respectively. The rest quasinormal modes can be realized as descendants of $\alpha_{\Delta}(X)$ and $\beta_{\Delta}(X)$. Though not explicitly spoken 
out in $[9,10]$, this claim actually relies on two facts: (a) $P_{i}$ preserves the equation of motion and (b) $P_{i}$ preserves the in-falling boundary condition near horizon. The former is obvious as we have seen at the end of previous section that the $\mathrm{SO}(1, d+1)$ action preserves uplift conditions and Pauli-Fierz conditions. The latter holds because $P_{i}$ is dominated by $-2 X_{i} \partial_{X^{+}}$near horizon, where $X_{i} \approx \Omega_{i}$ and $X^{+} \approx 2 e^{t-\rho}$. With the quasinormal modes known, we need to figure out the corresponding frequency. This is quite straightforward in our formalism. By construction, each quasinormal mode is an eigenfunction of the dilatation operator $D$, which is just $-\partial_{t}$ in the static patch. Using the in-falling boundary condition $e^{-i \omega_{\mathrm{QN}}(t-\rho)}$ near horizon, we can identify the scaling dimension, i.e. eigenvalue with respect to $D$, as $i \times$ (quasinormal frequency $\omega_{\mathrm{QN}}$ ). For example, a descendant of $\alpha_{\Delta}$ at level $n$ is a quasinormal mode of frequency $\omega_{\mathrm{QN}}=-i(\Delta+n)$ and similarly for $\beta_{\Delta}$.

To end the discussion about scalar quasinormal modes, let's compare our construction with the known result in literature. For example, in [7], the scalar quasinormal modes in $\mathrm{dS}_{d+1}$ are found to be

$$
\varphi_{\omega}^{\mathrm{QN}}(t, r, \Omega)=r^{\ell}\left(1-r^{2}\right)^{\frac{i \omega}{2}} F\left(\frac{\ell+i \omega+\Delta}{2}, \frac{\ell+i \omega+\bar{\Delta}}{2}, \frac{d}{2}+\ell, r^{2}\right) Y^{\ell \sigma}(\Omega) e^{-i \omega t}
$$

where $Y^{\ell \sigma}(\Omega)$ denote spherical harmonics on $S^{d-1}$ and the quasinormal frequency $\omega$ takes the following values

$$
\omega_{\ell, n}=-i(\Delta+\ell+2 n), \quad \bar{\omega}_{\ell, n}=-i(\bar{\Delta}+\ell+2 n), \quad \ell, n \in \mathbb{N}
$$

For fixed $\ell$ and $n$, the quasinormal modes of frequency $\omega_{\ell, n}$ or $\bar{\omega}_{\ell, n}$ have degeneracy $D_{\ell}^{d}$, i.e. the dimension of the spin- $\ell$ representation of $\mathrm{SO}(d)$. In particular, the two quasinormal modes corresponding to $\ell=n=0$ are

$$
\begin{aligned}
& \varphi_{\omega_{0,0}^{\mathrm{QN}}}^{\mathrm{QN}}=\frac{1}{\sqrt{A_{d-1}}} \frac{e^{-\Delta t}}{\left(1-r^{2}\right)^{\frac{\Delta}{2}}}=\frac{1}{\sqrt{A_{d-1}}}(\cosh \rho)^{\Delta} e^{-\Delta t} \\
& \varphi_{\bar{\omega}_{0,0} \mathrm{QN}}=\frac{1}{\sqrt{A_{d-1}}} \frac{e^{-\bar{\Delta} t}}{\left(1-r^{2}\right)^{\frac{\bar{\Delta}}{2}}}=\frac{1}{\sqrt{A_{d-1}}}(\cosh \rho)^{\bar{\Delta}} e^{-\bar{\Delta} t}
\end{aligned}
$$

where $A_{d-1}$ is the area of $S^{d-1}$. Apart from the normalization constant, these two quasinormal modes are exactly the primary quasinormal modes $\alpha_{\Delta}$ and $\beta_{\Delta}$ respectively. In addition to the match of the primary quasinormal modes, we can also show that the algebraic construction reproduces the quasinormal spectrum (3.6). Define $P=\sqrt{P_{i} P_{i}}$ and $\hat{P}_{i}=P^{-1} P_{i}$. Then the linear independent descendants of $\alpha_{\Delta}$ are of the form $P^{2 n+\ell} Y^{\ell \sigma}(\hat{P}) \alpha_{\Delta}$ with $\ell, n \in \mathbb{N}$. For fixed $\ell$ and $n$, these are quasinormal modes corresponding to $\omega_{\ell, n}$. Similarly $P^{2 n+\ell} Y^{\ell \sigma}(\hat{P}) \beta_{\Delta}$ represents quasinormal modes corresponding to $\bar{\omega}_{\ell, n}$.

\subsection{Massive higher spin fields}

As in the scalar case, we need to start from a solution of the Pauli-Fierz conditions (2.8)(2.10), subject to the tangential condition and homogeneous condition. A natural candidate 
is the higher spin boundary-to-bulk propagator. In AdS, the boundary-to-bulk propagator of a spin- $s$ field with a generic scaling dimension $\Delta(\neq d+s-2)$ is given by [31-33]

$$
K_{[\Delta, s]}^{\operatorname{AdS}}(X, U ; \xi, Z)=\frac{[(U \cdot Z)(\xi \cdot X)-(U \cdot \xi)(Z \cdot X)]^{s}}{(X \cdot \xi)^{\Delta+s}}
$$

where the null vector $\xi \in \mathbb{R}^{1, d+1}$ represents a boundary point and the null vector $Z \in \mathbb{C}^{1, d+1}$, satisfying $\xi \cdot Z=0$, encodes the boundary spin. $K_{[\Delta, s]}^{\mathrm{AdS}}$ in (3.8) scales like $K_{[\Delta, s]}^{\mathrm{AdS}}(\lambda X)=$ $\lambda^{-\Delta} K_{[\Delta, s]}^{\mathrm{AdS}}(X)$, which is the analogue of $\kappa=\Delta$ in eq. (2.7). In AdS, this scaling property corresponds to the choice of ordinary boundary condition. In $\mathrm{dS}$, on the other hand, both near-boundary fall-offs of a bulk field are dynamical and hence there are two boundary-tobulk propagators

$$
K_{[\kappa, s]}^{\mathrm{dS}}(X, U ; \xi, Z)=\frac{[(U \cdot Z)(\xi \cdot X)-(U \cdot \xi)(Z \cdot X)]^{s}}{(X \cdot \xi)^{\kappa+s}}
$$

where $\kappa \in\{\Delta, \bar{\Delta}\}$. Notice that $K_{i}$ in (2.24) does not involve any derivative with respect to $X^{+}$or $U^{+}$. So we can obtain primary mode functions from $K_{[\kappa, s]}^{\mathrm{dS}}(X, U ; \xi, Z)$ by putting $\xi^{A}$ at the southern pole of the past sphere, i.e. $\xi^{A}=(-1,0, \cdots, 0,1)$ and choosing $Z^{A}=$ $\left(0, z_{i}, 0\right)$, where $z_{i}$ itself is a null vector in $\mathbb{C}^{d}$ :

$$
\alpha_{[\Delta, s]}=\frac{\Phi^{s}}{\left(X^{+}\right)^{\Delta+s}}, \quad \beta_{[\Delta, s]}=\frac{\Phi^{s}}{\left(X^{+}\right)^{\bar{\Delta}+s}}
$$

where $\Phi=X^{+} u \cdot z-U^{+} x \cdot z$ (despite the lower case $x$ and $u$, indeed $x^{i} \equiv X^{i}$ and $\left.u^{i} \equiv U^{i}\right)$. $\alpha_{[\Delta, s]}$ and $\beta_{[\Delta, s]}$ are clearly primary quasinormal modes since in-falling boundary condition near horizon naturally follows from the lack of dependence on $X^{-}$and $U^{-}$. Given the two primary quasinormal modes, the whole quasinormal spectrum can be generated by acting $P_{i}$ on them repeatedly. In this sense, the algebraic construction of quasinormal modes for massive higher spin fields is a straightforward generalization of the scalar case. Before moving to massless higher spin fields, we want to emphasize that rigorously speaking, " $\alpha_{[\Delta, s]}$ " or " $\beta_{[\Delta, s]}$ " is not one primary quasinormal mode because varying $z^{i}$ would yield different quasinormal modes. Indeed, $\alpha_{[\Delta, s]}$ represents a collection of quasinormal modes with the same frequency and the vector space spanned by these quasinormal modes furnishes a spin- $s$ representation of $\mathrm{SO}(d)$. But for convenience, in most part of the paper, we will stick to the misnomer by calling, say $\alpha_{[\Delta, s]}$, the $\alpha$-mode.

\subsection{Massless higher spin fields}

When $\Delta$ hits $d-2+s$, i.e. the massless limit, $K_{[\Delta, s]}^{\text {AdS }}$ still holds as a boundary-to-bulk propagator in the so-called de Donder gauge [31, 32]. So we can extract de Sitter primary quasinormal modes, in de Donder gauge, from $K_{[\Delta, s]}^{\mathrm{AdS}}$ as in the massive case:

$$
\begin{array}{ll}
\alpha \text {-mode }: & \alpha^{(s)}(X, U ; z)=\frac{\Phi^{s}}{\left(X^{+}\right)^{2}}\left(\frac{R}{X^{+}}\right)^{d+2(s-2)} \\
\beta \text {-mode }: & \beta^{(s)}(X, U ; z)=\frac{\Phi^{s}}{\left(X^{+}\right)^{2}}
\end{array}
$$


where the $\mathrm{SO}(1, d+1)$-invariant $R=\sqrt{X^{2}}$ is inserted in $\alpha^{(s)},{ }^{4}$ so that it can have the same scaling property as $\beta^{(s)}$, which corresponds to $\kappa=2-s$ in (2.7). With this choice of $\kappa$, gauge transformation acts in the same way on both modes, schematically $\delta \alpha^{(s)}=U \cdot \partial_{X}(\cdots)$ and $\delta \beta^{(s)}=U \cdot \partial_{X}(\cdots)$.

Naively, one would expect (3.11) to be the end of story since we can generate the rest quasinormal modes as descendants of $\alpha^{(s)}$ and $\beta^{(s)}$, just as in the massive case. However, this expectation is only partially correct because, as we will show in the following, the quasinormal spectrum is significantly affected by gauge symmetry in the massless case compared to its massive counterpart. For example, the $\beta^{(s)}$-mode has quasinormal frequency $i \omega=2-s$, which would lead to an exponentially growing rather than damped behavior at future for $s \geq 3$. Gauge symmetry should be the only cure for this pathological growth and indeed, we do find that the $\beta$-mode is pure gauge for any $s \geq 1$ :

$$
\beta^{(s)}=U \cdot \partial_{X}\left(\xi_{s-1}\right), \quad \xi_{s-1}=\Phi^{s-1} \frac{x \cdot z}{X^{+}}
$$

where the gauge parameter $\xi_{s-1}$ can be realized as a descendant of another mode in the following sense:

$$
\xi_{s-1}=(z \cdot P) \eta_{s-1}, \quad \eta_{s-1}=-\frac{1}{2} \Phi^{s-1} \log X^{+}
$$

As a result, all the quasinormal modes in the $\beta$-tower are unphyical. The $\alpha$-mode itself is not pure gauge but it has some pure-gauge descendants:

$$
P \cdot \mathcal{D} \alpha^{(s)}=s(s+d-3) U \cdot \partial_{X}\left[\Phi^{s-1}\left(\frac{R}{X^{+}}\right)^{d+2 s-2}\right]
$$

where $\mathcal{D}_{i}=\left(\frac{d-2}{2}+z \cdot \partial_{z}\right) \partial_{z_{i}}-\frac{z_{i}}{2} \partial_{z}^{2}[34]$ strips off $z_{i}$ while respecting its nullness. If we write out the indices explicitly, (3.14) means $P_{i_{1}} \alpha_{i_{1} \cdots i_{s}}^{(s)}(X, U)=0$ up to gauge transformation, which is the reminiscence of a spin- $s$ conserved current.

In view of the discussion above, one might tend to claim that the quasinormal modes of a massless spin- $s$ field are $\alpha^{(s)}$ and its gauge invariant descendants. Granting this claim, the least damped quasinormal modes of photons and gravitons in $\mathrm{dS}_{d+1}$ have quasinormal frequency $i \omega=d-1$ and $i \omega=d$ respectively. However, this result is inconsistent with [7], where the author finds that the lowest quasinormal frequency of photons and gravitons is $i \omega=2 .^{5}$ The mismatch indicates that the $\alpha^{(s)}$-tower itself is not the end of the story and we have to modify our algebraic construction in the massless case to incorporate those missed quasinormal modes. The search of the hidden tower of gauge invariant quasinormal modes will be the task for the remaining part of this section.

\footnotetext{
${ }^{4}$ Including $R^{d+2(s-2)}$ does not spoil the Fierz-Pauli conditions (2.8)-(2.10).

${ }^{5}$ Here we have implicitly assumed $d \geq 4$. When $d=3$, the "tensor type" quasinormal modes of gravitational fluctuations in [7] do not exist because there are no transverse tensor harmonics on $S^{2}$. In this case, the lowest quasinormal frequency of a graviton is $i \omega=3$.
} 


\subsubsection{Maxwell fields}

The primary $\beta$-mode of a massless spin- 1 field is $\beta_{i}^{(1)}=\frac{\Phi_{i}}{\left(X^{+}\right)^{2}}$, where $\Phi_{i}=X^{+} u_{i}-U^{+} x_{i}$. It is pure gauge and the corresponding gauge parameter takes a very special form

$$
\beta_{i}^{(1)}=U \cdot \partial_{X}\left(P_{i} \eta_{0}\right)
$$

where $\eta_{0}$ is given by eq. (3.13) with $s=1$. Since $\mathfrak{s o}(1, d+1)$ action commutes with gauge transformation, we can switch the order of $P_{i}$ and $U \cdot \partial_{X}$ in $\beta_{i}^{(1)}$ :

$$
\beta_{i}^{(1)}=P_{i}\left(U \cdot \partial_{X} \eta_{0}\right)
$$

This new expression of $\beta_{i}^{(1)}$ inspires the following crucial observation. Treating $\beta_{i}^{(1)}$ as a vector field indexed by $i$ and treating $P_{i}$ as an ordinary derivative like $\partial_{i}$, then $\beta_{i}^{(1)}$ can be thought as a "pure gauge" mode with the gauge parameter being $U \cdot \partial_{X} \eta_{0}$. (We want to emphasize that this gauge symmetry structure is completely different from the bulk gauge symmetry, which takes the form $\delta(\cdots)=U \cdot \partial_{X}(\cdots)$. To distinguish it from the bulk gauge symmetry, we call it a "pseudo" gauge symmetry and its connection with the boundary gauge transformation will be discussed in section 4.2). Since $\beta_{i}^{(1)}$ is pure gauge with respect to the pseudo gauge symmetry, the (pseudo) field strength $\mathcal{F}_{i j} \equiv P_{i} \beta_{j}^{(1)}-P_{j} \beta_{i}^{(1)}$ vanishes identically. The vanishing of $\mathcal{F}_{i j}$ signals a potential way to obtain the new physical quasinormal modes, which will be implemented step by step as follows:

- First, we define a different $\beta$-mode $\hat{\beta}_{i}^{(1)}$ by deforming the scaling dimension of $\beta_{i}^{(1)}$ from 1 to $\Delta-1$ :

$$
\hat{\beta}_{i}^{(1)}(X, U) \equiv \frac{\Phi_{i}}{\left(X^{+}\right)^{\Delta}}=\left(X^{+}\right)^{2-\Delta} \beta_{i}^{(1)}, \quad \Delta \neq 2
$$

From the bulk field theory point of view, it amounts to giving a mass term to the Maxwell field to break the bulk $\mathrm{U}(1)$ gauge symmetry.

- Then the new pseudo field strength $\hat{\mathcal{F}}_{i j} \equiv P_{i} \hat{\beta}_{j}^{(1)}-P_{j} \hat{\beta}_{i}^{(1)}$ is nonvanishing

$$
\hat{\mathcal{F}}_{i j}=2(\Delta-2) \frac{x_{i} u_{j}-u_{i} x_{j}}{\left(X^{+}\right)^{\Delta}}
$$

- Stripping off the numerical factor $2(\Delta-2)$ and taking the limit $\Delta \rightarrow 2$ for the remaining part, we obtain a new non-pure-gauge mode function that is antisymmetric in $i, j$

$$
\gamma_{i j}^{(1)}(X, U) \equiv \frac{x_{i} u_{j}-u_{i} x_{j}}{\left(X^{+}\right)^{2}}=\frac{1}{2}\left(P_{i} \frac{u_{j}}{X^{+}}-P_{j} \frac{u_{i}}{X^{+}}\right)
$$

It is straightforward to check that $\gamma_{i j}^{(1)}$ satisfies all the requirements of being a quasinormal mode of frequency $i \omega=2$. Written in the form of (3.19), $\gamma_{i j}^{(1)}$ looks like a descendant of $\frac{u_{i}}{X^{+}}$. However, this descendant structure doesn't have any physical meaning because $\frac{u_{i}}{X^{+}}$ 
fails to satisfy the tangentiality condition (2.6). Actually, $\gamma_{i j}^{(1)}$ is a primary up to gauge transformation:

$$
K_{k} \gamma_{i j}^{(1)}=U \cdot \partial_{X}\left(\frac{\delta_{i k} x_{j}-\delta_{j k} x_{i}}{X^{+}}\right)
$$

Therefore, $\gamma_{i j}^{(1)}$ is a physical primary quasinormal mode and we will call the whole Verma module built from $\gamma_{i j}^{(1)}$ the " $\gamma$-tower" of quasinormal modes.

In the framework of pseudo gauge symmetry, $\gamma_{i j}^{(1)}$ can be thought as a U(1) field strength with $\frac{u_{i}}{X^{+}}$being the gauge potential. As a field strength, $\gamma_{i j}^{(1)}$ satisfies Bianchi identity $P_{[i} \gamma_{j k]}^{(1)}=0$ which imposes a nontrivial constraint on the descendants of $\gamma_{i j}^{(1)}$. When $d=3$, the field strength $\gamma_{i j}^{(1)}$ is dual to a vector $\tilde{\gamma}_{i}^{(1)}$ and the Bianchi identity is equivalent to a conservation equation $P_{i} \tilde{\gamma}_{i}^{(1)}=0$. In this case, the representation structure of the $\gamma$-tower is exactly the same as the $\alpha$-tower.

We will leave the comparison with intrinsic coordinate computation of quasinormal modes of Maxwell theory to appendix A.

\subsubsection{Linearized gravity}

$\mathbf{d} \geq 4$. The primary $\beta$-mode associated to a massless spin-2 field is $\beta^{(2)}=\frac{\Phi^{2}}{\left(X^{+}\right)^{2}}$. According to eq. (3.12) and (3.13), $\beta^{(2)}$ can be alternatively expressed as

$$
\beta_{i j}^{(2)}=P_{i}\left(U \cdot \partial_{X} \eta_{1}^{j}\right)+P_{j}\left(U \cdot \partial_{X} \eta_{1}^{i}\right)-\text { trace }
$$

where the null vectors $z$ in $\beta^{(2)}$ are stripped off. Treating $P_{i}$ as an ordinary derivative, the first two terms in $\beta_{i j}^{(2)}$ have the form of diffeomorphism transformation of (Euclidean) linearized gravity in $\mathbb{R}^{d}$. Given this pseudo diffeomorphism structure, we can naturally kill these two terms by considering the (pseudo) linearized Riemann tensor $R\left[\beta^{(2)}\right]_{i j k \ell}$, which is defined as

$$
R\left[\beta^{(2)}\right]_{i j k \ell} \equiv \frac{1}{2}\left(P_{j} P_{k} \beta_{i \ell}^{(2)}+P_{i} P_{\ell} \beta_{j k}^{(2)}-P_{i} P_{k} \beta_{j \ell}^{(2)}-P_{j} P_{\ell} \beta_{i k}^{(2)}\right)
$$

The remaining pure trace term in $\beta_{i j}^{(2)}$ drops out by projecting $R\left[\beta^{(2)}\right]_{i j k \ell}$ to the "linearized Weyl tensor" $\mathcal{C}\left[\beta^{(2)}\right]_{i j k \ell}$ which is defined as the traceless part $R\left[\beta^{(2)}\right]_{i j k \ell}$ and carries the $\square$ representation of $\mathrm{SO}(d)$. By construction, $\mathcal{C}\left[\beta^{(2)}\right]_{i j k \ell}$ would vanish just as the $\mathrm{U}(1)$ field strength $\mathcal{F}_{i j}$ in the spin-1 case. Due to this similarity, it is quite natural to expect that the new spin-2 physical primary quasinormal mode will be produced by the same "deformation+ limiting" procedure, whose steps are listed here again for reader's convenience: (a) deform the $\beta^{(2)}$ mode by sending it to $\hat{\beta}^{(2)} \equiv \frac{\Phi^{2}}{\left(X^{+}\right)^{\Delta}}=\left(X^{+}\right)^{2-\Delta} \beta^{(2)},(b)$ compute the Weyl tensor $\mathcal{C}\left[\hat{\beta}^{(2)}\right]_{i j k \ell}$ associated to $\hat{\beta}^{(2)},(c)$ strip off the overall factor $(\Delta-2)$ in $\mathcal{C}\left[\hat{\beta}^{(2)}\right]_{i j k \ell}$ and take the limit $\Delta \rightarrow 2$ for the remaining part. To show the limiting procedure $(c)$ more explicitly, expand, for instance, the first term in $R\left[\hat{\beta}^{(2)}\right]$ :

$$
P_{j} P_{k} \hat{\beta}_{i \ell}^{(2)}=2(\Delta-2) \beta_{i \ell}^{(2)} P_{j} \frac{x_{k}}{\left(X^{+}\right)^{\Delta-1}}+2(\Delta-2) \frac{x_{(k} P_{j} \beta_{i \ell}^{(2)}}{\left(X^{+}\right)^{\Delta-1}}+\frac{P_{j} P_{k} \beta_{i \ell}^{(2)}}{\left(X^{+}\right)^{\Delta-2}}
$$


where the convention for symmetrization is $x_{(k} P_{j)}=x_{k} P_{j}+x_{j} P_{k}$. The last term in (3.23) does not contribute to the Weyl tensor $\mathcal{C}\left[\hat{\beta}^{(2)}\right]$ because the first two terms of $\beta_{i \ell}^{(2)}$ take the form of gauge transformation and the pure trace part is explicitly subtracted out by construction of Weyl tensor. So we will drop it henceforth. The remaining terms are proportional to $\Delta-2$, so the limiting procedure is applicable to them

$$
\begin{aligned}
\lim _{\Delta \rightarrow 2} \frac{P_{j} P_{k} \hat{\beta}_{i \ell}^{(2)}}{\Delta-2} & =P_{j}\left(\frac{2 x_{k}}{X^{+}}\right) \beta_{i \ell}^{(2)}+\frac{2 x_{k}}{X^{+}} P_{j} \beta_{i \ell}^{(2)}+\frac{2 x_{j}}{X^{+}} P_{k} \beta_{i \ell}^{(2)} \\
& =P_{j} P_{k}\left(-\log \left(X^{+}\right) \beta_{i \ell}^{(2)}\right)+\log \left(X^{+}\right) P_{j} P_{k} \beta_{i \ell}^{(2)}
\end{aligned}
$$

where the last term drops out from Weyl tensor $\mathcal{C}\left[\hat{\beta}^{(2)}\right]$ for the same reason given above. Therefore the new physical quasinormal mode is schematically

$$
\gamma_{i j k \ell}^{(2)}(X, U) \equiv \mathcal{C}[h]_{i j k \ell}, \quad h_{i j}=-\log \left(X^{+}\right) \beta_{i j}^{(2)}
$$

which has scaling dimension 2 (or quasinormal frequency $i \omega=2$ ) and carries the representation of $\mathrm{SO}(d)$. The primariness of $\gamma_{i j k \ell}^{(2)}$ is proved in appendix C.

$\mathbf{d}=3$. The $d=3$ case is degenerate and requires a separate discussion because the $3 \mathrm{D}$ Weyl tensor $\mathcal{C}\left[\hat{\beta}^{(2)}\right]_{i j k \ell}$ vanishes identically for arbitrary choice of $\Delta$. So the deformation and limiting procedure used above fails to yield any quasinormal mode when $d=3$. The solution to this problem is using Cotton tensor, the 3D analogue of Weyl tensor. On a 3 -dimensional Riemann manifold with metric $g_{i j}$, the Cotton tensor is given by $[17,18]$

$$
\mathcal{C}_{i}^{j}[g]=\nabla_{k}\left(R_{i \ell}-\frac{1}{4} R g_{i \ell}\right) \epsilon^{k \ell j}
$$

and the vanishing of Cotton tensor is the necessary and sufficient condition for the 3dimensional manifold to be conformally flat. (In spite of the abuse of notation $\mathcal{C}$, it will be clear from the context that $\mathcal{C}$ means Weyl tensor when $d \geq 4$ and means Cotton tensor when $d=3$ ). At the linearized level, i.e. $g_{i j}=\delta_{i j}+\phi_{i j}$, the Cotton tensor becomes

$$
\mathcal{C}[\phi]_{i j}=\partial_{k} R_{i \ell}[\phi] \epsilon_{k \ell j}-\frac{1}{4} \epsilon_{k i j} \partial_{k} R[\phi]
$$

where $R[\phi]_{i j}, R[\phi]$ are linearized Ricci tensor and linearized Ricci scalar respectively. In addition, the linearized Cotton tensor is actually symmetric in $i, j$ which can be checked by contracting it with $\epsilon_{i j m}$

$$
\mathcal{C}[\phi]_{i j} \epsilon_{i j m}=\frac{1}{2} \partial_{m} R[\phi]-\partial_{i} R_{i m}=0
$$

where the last step is a well-known result of Bianchi identity of Riemann tensor. Since $\mathcal{C}[\phi]_{i j}$ is symmetric and traceless, it will be convenient to restore the null vector $z$

$$
\mathcal{C}[\phi ; z]=\partial_{k} R_{i \ell}[\phi] \epsilon_{k \ell j} z_{i} z_{j}=\frac{1}{2} \epsilon_{k \ell j}\left(\partial_{i} \partial_{k} \partial_{m} \phi_{m \ell}-\partial^{2} \partial_{k} \phi_{i \ell}\right) z_{i} z_{j}
$$


In the context of quasinormal modes, we can similarly construct a pseudo Cotton tensor with ordinary derivative $\partial_{i}$ in eq. (3.29) replaced by momentum operator $P_{i}$

$$
\mathcal{C}[\phi ; z]=\frac{1}{2} \epsilon_{k \ell j}\left(P_{i} P_{k} P_{m} \phi_{m \ell}-P^{2} P_{k} \phi_{i \ell}\right) z_{i} z_{j}
$$

Because Cotton tensor is invariant under diffeomorphism and local Weyl transformation by construction, $\mathcal{C}[\phi ; z]$ vanishes exactly when $\phi=\beta^{(2)}$. As a result, applying the deformation and limiting procedure to the Cotton tensor $\mathcal{C}\left[\beta^{(2)} ; z\right]$ would yield a new physical primary quasinormal mode

$$
\gamma^{(2)}(X, U ; z)=\frac{z \cdot(x \wedge u)}{\left(X^{+}\right)^{3}}\left(X^{+} U^{-} x \cdot z-X^{+} X^{-} u \cdot z+x^{2} u \cdot z-(u \cdot x)(x \cdot z)\right)
$$

where $(x \wedge u)_{i}=\epsilon_{i j k} x_{j} u_{k}$. Compared to higher dimensional cases, $\gamma^{(2)}$ in $d=3$ is different mainly in two ways: $(a)$ it has scaling dimension 3 because Cotton tensor involves three derivatives while Weyl tensor only involves $2,(b)$ it carries a spin-2 representation of $\mathrm{SO}(3)$ and the Bianchi identity in higher dimension becomes a "conservation" equation $P_{i} \gamma_{i j}^{(2)}=0$. Due to these two properties, the $\gamma^{(2)}$-tower of quasinormal modes in $\mathrm{dS}_{4}$ is isomorphic to the $\alpha^{(2)}$-tower.

\subsubsection{Massless higher spin fields}

With the spin-1 and spin-2 examples worked out explicitly, we will continue to show that for any massless higher spin field, there exist the primary $\gamma$-mode. For a massless spin- $s$ field, the primary $\beta$-mode given by eq. (3.12) and (3.13) can be written as

$$
\beta_{i_{1} \cdots i_{s}}^{(s)}=P^{\left(i_{1}\right.} U \cdot \partial_{X} \eta_{s-1}^{\left.i_{2} \cdots i_{s}\right)}-\text { trace }
$$

Treating $P^{i}$ as an ordinary derivative, apart from the pure trace part, $\beta^{(s)}$ has the form of gauge transformation of $d$-dimensional linearized spin- $s$ gravity. Such a gauge transformation can be eliminated by using the higher spin Riemann tensor

$$
R[\phi]_{i_{1} \ell_{1}, \cdots, i_{s} \ell_{s}} \equiv \Pi_{s s} P_{i_{1}} \cdots P_{i_{s}} \phi_{\ell_{1} \cdots \ell_{s}}
$$

where $\Pi_{s s}$ is a projection operator ensuring $R[\phi]_{i_{1} \ell_{1}, \cdots, i_{s} \ell_{s}}$ carries the $\mathbb{Y}_{s s}$ representation of $\operatorname{GL}(d, \mathbb{R})$. ( $\mathbb{Y}_{n m}$ denotes a 2-row Young diagram with $n$ boxes in the first row and $m$ boxes in the second. When $m=0$, we use $\mathbb{Y}_{n}$.) More explicitly, $\Pi_{s s}$ can be realized by antisymmetrizing the $s$ pairs of indices: $\left[i_{1}, \ell_{1}\right], \cdots,\left[i_{s}, \ell_{s}\right][19,35]$. The higher spin Weyl tensor $\mathcal{C}[\phi]_{i_{1} \ell_{1}, \cdots, i_{s} \ell_{s}}$ is defined as the traceless part of $R[\phi]_{i_{1} \ell_{1}, \cdots, i_{s} \ell_{s}}$ and thus it carries the $\mathbb{Y}_{s s}$ representation of $\mathrm{SO}(d) .{ }^{6}$ Since Weyl tensor is invariant under diffeomorphism and

\footnotetext{
${ }^{6}$ For simplicity, we assume $d \geq 4$ so the higher spin Weyl tensor is nonvanishing. When $d=3$, we should use higher spin Cotton tensor $\mathcal{C}_{i_{1} \cdots i_{s}}[19-21]$ that is symmetric and traceless. The Bianchi identity for Cotton tensor is a conservation equation $P_{i_{1}} \mathcal{C}_{i_{1} \cdots i_{s}}=0$. In addition, the definition of Cotton tensor involves $2 s-1$ momentum operators and hence the associated primary $\gamma$-mode would have scaling dimension $1+s$ instead of 2 .
} 
local Weyl transformation, $\mathcal{C}\left[\beta^{(s)}\right]$ vanishes exactly. Thus we can apply the deformation procedure to it and obtain the following quasinormal mode

$$
\gamma_{i_{1} \ell_{1}, \cdots i_{s} \ell_{s}}^{(s)}(X, U) \equiv \mathcal{C}\left[h^{(s)}\right]_{i_{1} \ell_{1}, \cdots, i_{s} \ell_{s}}, \quad h_{\ell_{1} \cdots \ell_{s}}^{(s)}=-\log \left(X^{+}\right) \beta_{\ell_{1} \cdots \ell_{s}}^{(s)}
$$

In appendix C, we show that $\gamma_{i_{1} \ell_{1}, \cdots, i_{s} \ell_{s}}^{(s)}$ represents $D_{s s}^{d}$ primary quasinormal modes of scaling dimension 2 that carry $\mathbb{Y}_{s s}$ representation of $\mathrm{SO}(d)$, where $D_{s s}^{d}$ is the dimension of the $\mathbb{Y}_{s s}$ representation. (We will use $D_{n m}^{d}$ for the dimension of $\mathbb{Y}_{n m}$ representation of $\mathrm{SO}(d)$ and $D_{n}^{d}$ for the dimension of $\mathbb{Y}_{n}$ representation.) In the same appendix, we also show that these primary quasinormal modes can alternatively be expressed in the following form

$$
\gamma^{(s)}(X, U)=\frac{T_{s s}(x, u)}{\left(X^{+}\right)^{2}}
$$

where $T_{s s}(x, u)$ is a homogeneous polynomial in both $x$ and $u$ of degree $s$ and satisfies (C.10). The space of such $T_{s s}$ carries the $\mathbb{Y}_{s s}$ representation of $\operatorname{SO}(d)$ [36]. One obvious example of $T_{s s}$ is

$$
T_{s s}(x, u)=\left[\left(x^{1}+i x^{2}\right)\left(u^{3}+i u^{4}\right)-\left(x^{3}+i x^{4}\right)\left(u^{1}+i u^{2}\right)\right]^{s}
$$

In the representation language, the example given by eq. (3.36) is actually the lowest-weight state in $\mathbb{Y}_{s s}$. Therefore, we are able to generate the whole $\gamma$-tower by acting $P_{i}$ and $M_{i j}$ on the following quasinormal mode:

$$
\gamma_{l w}^{(s)}(X, U)=\frac{\left[\left(X^{1}+i X^{2}\right)\left(U^{3}+i U^{4}\right)-\left(X^{3}+i X^{4}\right)\left(U^{1}+i U^{2}\right)\right]^{s}}{\left(X^{+}\right)^{2}}
$$

This is a strikingly universal expression that works for any $s \geq 1$ and $d \geq 4$. In static patch coordinate, the nonvanishing components of (3.37) are

$$
\gamma_{l w, a_{1} \cdots a_{s}}^{(s)}(t, r, \Omega)=\frac{r^{2 s} e^{-2 t}}{\left(1-r^{2}\right)}\left(\Omega_{12} \partial_{\vartheta^{a_{1}}} \Omega_{34}-\Omega_{34} \partial_{\vartheta^{a_{1}}} \Omega_{12}\right) \cdots\left(\Omega_{12} \partial_{\vartheta^{a_{s}}} \Omega_{34}-\Omega_{34} \partial_{\vartheta^{a_{s}}} \Omega_{12}\right)
$$

where $\Omega_{12}=\Omega_{1}+i \Omega_{2}$ and $\Omega_{34}=\Omega_{3}+i \Omega_{4}$. One can check that the $\Omega$-dependent part of (3.38) is actually a divergence-free spin- $s$ tensor harmonics on $S^{d-1}$. This is also expected from the representation side because these tensor harmonics also furnish the $\mathbb{Y}_{s s}$ representation of $\mathrm{SO}(d)$.

For the completeness of the final result, we also give the unique lowest-weight state in the $\alpha^{(s)}$-tower here

$$
\alpha_{l w}^{(s)}(X, U)=\frac{\left[X^{+}\left(U^{1}+i U^{2}\right)-U^{+}\left(X^{1}+i X^{2}\right)\right]^{s}}{\left(X^{+}\right)^{2}}\left(\frac{R}{X^{+}}\right)^{d+2(s-2)}
$$

Then all the quasinormal modes are built from $\alpha_{l w}^{(s)}$ (cf. (3.39)) and $\gamma_{l w}^{(s)}$ (cf. (3.37)) with the action of $P_{i}$ and $M_{i j}$. 


\section{Quasinormal modes from a QFT point of view}

In the previous section, we presented a pure algebraic method to construct quasinormal modes of scalars and higher spin fields. In this section, we will provide a simple physical picture for this method from a bulk QFT point of view. In particular, we will use scalar fields and Maxwell fields to illustrate this intuitive picture explicitly and then give a brief comment on general massless higher spin fields. Through out this section, the bulk quantum fields are defined in the southern past planar coordinate $\left(\eta, y^{i}\right)$ of $\mathrm{dS}_{d+1}$ (the region "S"+"P" in figure 1):

$$
X^{0}=\frac{1+y^{2}-\eta^{2}}{2 \eta}, \quad X^{i}=-\frac{y^{i}}{\eta}, \quad X^{d+1}=-\frac{1-y^{2}+\eta^{2}}{2 \eta}
$$

where $\eta<0$ and the quasinormal modes are still defined in the southern static patch, which corresponds to $y<-\eta$ in eq. (4.1).

\subsection{Scalar fields}

Let $\varphi$ be a scalar field of scaling dimension $\Delta$. Near the past boundary, it has the following asymptotic behavior

$$
\varphi(\eta, y) \approx(-\eta)^{\Delta} \mathcal{O}^{(\alpha)}(y)+(-\eta)^{\bar{\Delta}} \mathcal{O}^{(\beta)}(y)
$$

Define quantum operators $\mathcal{L}_{A B}$ such that $L_{A B} \varphi=-\left[\mathcal{L}_{A B}, \varphi\right]$. Then $\mathcal{O}^{(\alpha)}$ and $\mathcal{O}^{(\beta)}$ are primary operators in the sense that

$$
\begin{array}{llrl}
{\left[\mathcal{D}, \mathcal{O}^{(\alpha)}(0)\right]} & =\Delta \mathcal{O}^{(\alpha)}(0), & & {\left[\mathcal{K}_{i}, \mathcal{O}^{(\alpha)}(0)\right]=0} \\
{\left[\mathcal{D}, \mathcal{O}^{(\beta)}(0)\right]} & =\bar{\Delta} \mathcal{O}^{(\beta)}(0), & & {\left[\mathcal{K}_{i}, \mathcal{O}^{(\beta)}(0)\right]=0}
\end{array}
$$

The bulk two-point function of $\varphi$ defined with respect to the Euclidean vacuum $|E\rangle$ is given by [37]

$$
\left\langle E\left|\varphi(X) \varphi\left(X^{\prime}\right)\right| E\right\rangle=\frac{\Gamma(\Delta) \Gamma(\bar{\Delta})}{(4 \pi)^{\frac{d+1}{2}} \Gamma\left(\frac{d+1}{2}\right)} F\left(\Delta, \bar{\Delta}, \frac{d+1}{2}, \frac{1+P}{2}\right)
$$

where $P=X \cdot X^{\prime}$. We push $X^{\prime}$ to the past southern pole, i.e. $y^{\prime i}=0$ and $\eta^{\prime} \rightarrow 0^{-}$, then $P$ is approximately $-\frac{X^{+}}{2 \eta^{\prime}} \rightarrow \infty$. For $P \rightarrow \infty$, the hypergeometric function in (4.4) has two leading asymptotic behaviors: $P^{-\Delta}$ and $P^{-\bar{\Delta}}$. Schematically, it means

$$
\left\langle E\left|\varphi(X) \varphi\left(\eta^{\prime} \rightarrow 0, y^{\prime i}=0\right)\right| E\right\rangle \approx c_{\Delta} \frac{\left(-\eta^{\prime}\right)^{\Delta}}{\left(X^{+}\right)^{\Delta}}+c_{\bar{\Delta}} \frac{\left(-\eta^{\prime}\right)^{\bar{\Delta}}}{\left(X^{+}\right)^{\bar{\Delta}}}
$$

where $c_{\Delta}$ and $c_{\bar{\Delta}}$ are two known constants. Comparing the eq. (4.2) and eq. (4.5), we find that $\left\langle E\left|\varphi(X) \mathcal{O}^{(\alpha)}(0)\right| E\right\rangle$ produces the primary quasinormal mode $\alpha_{\Delta}(X)$ and $\left\langle E\left|\varphi(X) \mathcal{O}^{(\beta)}(0)\right| E\right\rangle$ produces the primary quasinormal mode $\beta_{\Delta}(X)$. Altogether, the scalar primary quasinormal modes in southern static patch can be produced by inserting primary operator $\mathcal{O}^{(\alpha)}$ or $\mathcal{O}^{(\beta)}$ at the southern pole of the past sphere and other quasinormal modes can be produced by inserting descendants of $\mathcal{O}^{(\alpha)}$ or $\mathcal{O}^{(\beta)}$. 


\subsection{Maxwell fields}

We want to derive the two primary quasinormal modes of Maxwell field in $\mathrm{dS}_{4}$ using local operators. First, let's pull back $\alpha_{i}^{(1)}$ and $\gamma_{i j}^{(1)}$ to planar patch:

$$
\alpha_{i, \mu}^{(1)}=\frac{\partial X^{A}}{\partial y^{\mu}} \partial_{U^{A}} \alpha_{i}^{(1)}, \quad \gamma_{i j, \mu}^{(1)}=\frac{\partial X^{A}}{\partial y^{\mu}} \partial_{U^{A}} \gamma_{i j}^{(1)}
$$

where $y^{\mu}=\left(\eta, y^{i}\right)$ are planar patch coordinates defined in eq. (4.1).

\section{The $\alpha$-mode:}

$$
\alpha_{i, \eta}^{(1)}=\frac{2 y_{i} \eta^{2}}{\left(\eta^{2}-y^{2}\right)^{3}}, \quad \alpha_{i, j}^{(1)}=-\frac{\eta\left(2 y_{i} y_{j}+\delta_{i j}\left(\eta^{2}-y^{2}\right)\right)}{\left(\eta^{2}-y^{2}\right)^{3}}
$$

For later convenience, we perform a gauge transformation to kill the timelike component, which can be done by choosing the following gauge parameter ${ }^{7}$

$$
\xi=\frac{y_{i}}{4 y^{3}}\left(\frac{y \eta\left(\eta^{2}+y^{2}\right)}{\left(\eta^{2}-y^{2}\right)^{2}}-\frac{1}{2} \log \frac{\eta+y}{\eta-y}\right)
$$

The resulting spatial part of $\alpha_{i}^{(1)}$ becomes

$$
\tilde{\alpha}_{i, j}^{(1)}=\left(\partial_{y^{i}} \partial_{y^{j}}-\delta_{i j} \partial_{y}^{2}\right) \frac{\log \frac{\eta+y}{\eta-y}}{8 y}
$$

The $\gamma$-mode:

$$
\gamma_{i j, \eta}^{(1)}=0, \quad \gamma_{i j, k}^{(1)}=\frac{y_{i} \delta_{j k}-y_{j} \delta_{i k}}{\left(\eta^{2}-y^{2}\right)^{2}}
$$

The timelike component is automatically vanishing.

Next, we do a mode expansion for a Maxwell field $A_{\mu}$ in the Coulomb gauge [38]:

$$
A_{i}(\eta, y)=-\int \frac{d^{3} k}{(2 \pi)^{3}}\left(\mathcal{O}_{i}^{(\alpha)}(k) \frac{\sin (k \eta)}{k}-\mathcal{O}_{i}^{(\beta)}(k) \cos (k \eta)\right) e^{i k \cdot y}
$$

where the two primary operators $\mathcal{O}_{i}^{(\alpha)}, \mathcal{O}_{i}^{(\beta)}$ capture the leading asymptotic behavior of $A_{i}$ near the past boundary

$$
A_{i}\left(\eta \rightarrow 0^{-}, y\right) \approx(-\eta) \mathcal{O}_{i}^{(\alpha)}(y)+\mathcal{O}_{i}^{(\beta)}(y)
$$

They also satisfy the following vacuum two-point functions in momentum space:

$$
\begin{aligned}
\left\langle E\left|\mathcal{O}_{i}^{(\beta)}(k) \mathcal{O}_{j}^{(\beta)}\left(k^{\prime}\right)\right| E\right\rangle & =\frac{1}{2 k}\left(\delta_{i j}-\frac{k_{i} k_{j}}{k^{2}}\right)(2 \pi)^{3} \delta^{3}\left(k+k^{\prime}\right) \\
\left\langle E\left|\mathcal{O}_{i}^{(\alpha)}(k) \mathcal{O}_{j}^{(\beta)}\left(k^{\prime}\right)\right| E\right\rangle & =\frac{i}{2}\left(\delta_{i j}-\frac{k_{i} k_{j}}{k^{2}}\right)(2 \pi)^{3} \delta^{3}\left(k+k^{\prime}\right) \\
\left\langle E\left|\mathcal{O}_{i}^{(\alpha)}(k) \mathcal{O}_{j}^{(\alpha)}\left(k^{\prime}\right)\right| E\right\rangle & =\frac{k}{2}\left(\delta_{i j}-\frac{k_{i} k_{j}}{k^{2}}\right)(2 \pi)^{3} \delta^{3}\left(k+k^{\prime}\right) \\
\left\langle E\left|\mathcal{O}_{i}^{(\beta)}(k) \mathcal{O}_{j}^{(\alpha)}\left(k^{\prime}\right)\right| E\right\rangle & =-\frac{i}{2}\left(\delta_{i j}-\frac{k_{i} k_{j}}{k^{2}}\right)(2 \pi)^{3} \delta^{3}\left(k+k^{\prime}\right)
\end{aligned}
$$

\footnotetext{
${ }^{7}$ Since quasinormal modes are still defined in the static patch, which corresponds to $\eta+y<0$, we are away from the branch cut of logarithm and the gauge parameter is real.
} 
Like in the scalar case, we insert the primary operator $\mathcal{O}_{i}^{(\alpha)}$ at the southern pole of the past sphere and it produces a mode in the bulk:

$$
\begin{aligned}
\left\langle E\left|A_{i}(\eta, y) \mathcal{O}_{j}^{(\alpha)}(0)\right| E\right\rangle & =-\frac{i}{2} \int \frac{d^{3} k}{(2 \pi)^{3}}\left(\delta_{i j}-\frac{k_{i} k_{j}}{k^{2}}\right) e^{i k \cdot y-i k \eta} \\
& =-\frac{i}{4 \pi^{2}}\left(\partial_{y^{i}} \partial_{y^{j}}-\delta_{i j} \partial_{y}^{2}\right) \int_{0}^{\infty} \frac{d k}{k y} \sin (k) e^{-i \frac{\eta}{y} k}
\end{aligned}
$$

where the integral over $k$ depends on the relative size of $y$ and $-\eta$ because $^{8}$

$$
\int_{0}^{\infty} \frac{d k}{k} \sin (k) e^{i a k}= \begin{cases}\frac{i}{2} \log \frac{a+1}{a-1}, & |a|>1 \\ \frac{\pi}{2}+\frac{i}{2} \log \frac{1+a}{1-a}, & |a|<1\end{cases}
$$

In a physical picture, the jumping at $|a|=1$ reflects horizon crossing. For quasinormal modes defined in southern static patch, which has $y<-\eta$, the $k$-integral in (4.14) corresponds to the top case of (4.15):

$$
\left\langle E\left|A_{i}(\eta, y) \mathcal{O}_{j}^{(\alpha)}(0)\right| E\right\rangle=\left(\partial_{y^{i}} \partial_{y^{j}}-\delta_{i j} \partial_{y}^{2}\right)\left(\frac{-1}{8 \pi^{2} y} \log \frac{\eta+y}{\eta-y}\right)
$$

Up to normalization, we precisely reproduce the $\alpha_{i}^{(1)}$ quasinormal mode given by (4.9). Similarly, we can insert the $\mathcal{O}_{i}^{(\beta)}$ at the southern pole of the past sphere and it yields

$$
\left\langle E\left|A_{k}(\eta, y) \mathcal{O}_{i}^{(\beta)}(0)\right| E\right\rangle=\int \frac{d^{3} k}{(2 \pi)^{3}}\left(\delta_{i k}-\frac{k_{i} k_{k}}{k^{2}}\right) \frac{e^{i k \cdot y-i k \eta}}{2 k}
$$

Due to the extra $\frac{1}{k}$ in the integrand compared to eq. (4.14), this mode suffers from an IR divergence around $k=0$. However, this divergence can be eliminated if we replace $\mathcal{O}_{i}^{(\beta)}(0)$ by a "curvature" $\mathcal{O}_{i j}^{(\gamma)}(0) \equiv P_{i} \mathcal{O}_{j}^{(\beta)}(0)-P_{j} \mathcal{O}_{i}^{(\beta)}(0)$ as in the construction of $\gamma_{i j}^{(1)}$. The insertion of $\mathcal{O}_{i}^{(\gamma)}$ at southern pole yields

$$
\left\langle E\left|A_{k}(\eta, y) \mathcal{O}_{i j}^{(\gamma)}(0)\right| E\right\rangle=\frac{i}{2} \int \frac{d^{3} k}{(2 \pi)^{3}} \frac{k_{i} \delta_{j k}-k_{j} \delta_{i k}}{k} e^{i k \cdot y-i k \eta}=\frac{1}{2 \pi^{2}} \frac{y_{j} \delta_{i k}-y_{i} \delta_{j k}}{\left(\eta^{2}-y^{2}\right)^{2}}
$$

which is exactly the $\gamma_{i j}^{(1)}$ quasinormal mode, cf. (4.10), up to normalization. Note that in the definition of $\mathcal{O}_{i j}^{(\gamma)}$, we implicitly use the pseudo gauge symmetry structure. On the other hand, $P_{i}$ becomes the ordinary derivative $\partial_{i}$ at boundary and hence $\mathcal{O}_{i j}^{(\gamma)}$ is indeed the curvature corresponding to the boundary gauge symmetry. Therefore, the classically pseudo gauge symmetry can be identified as the boundary gauge symmetry in quantum theory. In this sense, $\mathcal{O}_{i j}^{(\gamma)} \sim \epsilon_{i j k} B_{k}$ has the interpretation as a boundary magnetic field and $\mathcal{O}_{i}^{(\alpha)} \sim E_{i}$ has the interpretation as a boundary electric field, subject to the constraint $\nabla \cdot E=0$. So the quasinormal modes of Maxwell fields are produced by

\footnotetext{
${ }^{8}$ The two cases can be uniformly treated if we give $a$ a small positive imaginary part, i.e. $a \rightarrow a+i \epsilon, \epsilon>0$. With this $i \epsilon$ prescription, $\frac{i}{2} \log \frac{a+i \epsilon+1}{a+i \epsilon-1}$ works for both cases. In bulk, it amounts to Wick rotating the planar coordinate time: $\eta \rightarrow e^{i \epsilon} \eta$.
} 
the electric/magnetic field operator, together with their derivatives, inserted at the past southern pole, cf. figure 1.

In general, a free massless higher spin field $\varphi_{\mu_{1} \cdots \mu_{s}}$ in a suitable gauge has the following asymptotic behavior near the past boundary

$$
\varphi_{i_{1} \cdots i_{s}}\left(\eta \rightarrow 0^{-}, y\right) \approx(-\eta)^{d-2} \mathcal{O}_{i_{1} \cdots i_{s}}^{(\alpha)}(y)+(-\eta)^{2-2 s} \mathcal{O}_{i_{1} \cdots i_{s}}^{(\beta)}(y)
$$

where $\mathcal{O}_{i_{1} \cdots i_{s}}^{(\alpha)}(y)$ is a gauge-invariant boundary conserved current and $\mathcal{O}_{i_{1} \cdots i_{s}}^{(\beta)}(y)$ is a boundary gauge field. ${ }^{9}$ From $\mathcal{O}_{i_{1} \cdots i_{s}}^{(\beta)}(y)$, we can build a boundary Weyl tensor $\mathcal{O}_{i_{1} j_{1}, \cdots, i_{s} j_{s}}^{(\gamma)}$ that is gauge invariant. Then inserting operators in the conformal family of $\mathcal{O}_{i_{1} \cdots i_{s}}^{(\alpha)}$ at the past southern pole produces the $\alpha$-tower of quasinormal modes and inserting operators in the conformal family of $\mathcal{O}_{i_{1} j_{1}, \cdots, i_{s} j_{s}}^{(\gamma)}$ at the past southern pole produces the $\gamma$-tower of quasinormal modes

\section{Quasinormal modes and $\mathrm{SO}(1, d+1)$ characters}

In the section 3, we describe a procedure to construct quasinormal modes of scalar fields and higher spin fields in $\mathrm{dS}_{d+1}$. In this section, we will extract the whole quasinormal spectrum by using this construction and show that it's related to the Harish-Chandra group character of $\mathrm{SO}(1, d+1)$. To collect the information of quasinormal modes of certain field $\phi$ in a compact expression, we define a "quasinormal character":

$$
\chi_{\phi}^{\mathrm{QN}}(q)=\sum_{\omega} d_{\omega} q^{i \omega}, \quad 0<q<1
$$

where the sum runs over all quasinormal frequencies of $\phi$ and $d_{\omega}$ is the degeneracy of quasinormal modes with frequency $\omega$. Due to the representation structure of the quasinormal modes, the quasinormal character $\chi_{\phi}^{\mathrm{QN}}(q)$ naturally splits into two different parts, with each part involves either the $\alpha$-tower or $\beta / \gamma$-tower of quasinormal modes. For example, let $\phi$ be a real scalar field of scaling dimension $\Delta$. The quasinormal modes in the $\alpha$-tower have frequencies $i \omega_{n}^{\alpha}=\Delta+n, n \geq 0$ and for each $n$ the degeneracy is $\left(\begin{array}{c}d+n-1 \\ d-1\end{array}\right)$. Thus the $\alpha$-part of the quasinormal character is

$$
\chi_{\Delta}^{\mathrm{QN}, \alpha}(q)=\sum_{n \geq 0}\left(\begin{array}{c}
d+n-1 \\
d-1
\end{array}\right) q^{\Delta+n}=\frac{q^{\Delta}}{(1-q)^{d}}
$$

Similarly, the contribution of $\beta$-tower is $\chi_{\Delta}^{\mathrm{QN}, \beta}(q)=\frac{q^{\bar{\Delta}}}{(1-q)^{d}}$. Altogether, we obtain the full quasinormal character of $\phi$

$$
\chi_{\Delta}^{\mathrm{QN}}(q)=\chi_{\Delta}^{\mathrm{QN}, \alpha}(q)+\chi_{\Delta}^{\mathrm{QN}, \beta}(q)=\frac{q^{\Delta}+q^{\bar{\Delta}}}{(1-q)^{d}}
$$

\footnotetext{
${ }^{9}$ For a bulk gauge transformation $\delta \varphi_{\mu_{1} \cdots \mu_{s}}=\nabla_{\left(\mu_{1}\right.} \xi_{\left.\mu_{2} \cdots \mu_{s}\right)}$, the asymptotic behavior of $\xi$ near the past boundary is $\xi_{i_{1} \cdots i_{s}}(\eta, y) \approx(-\eta)^{d} A_{i_{1} \cdots i_{s-1}}(y)+(-\eta)^{2-2 s} B_{i_{1} \cdots i_{s-1}}(y)$. $\mathcal{O}_{i_{1} \cdots i_{s}}^{(\alpha)}$ is clearly invariant under this transformation as the $A$-mode falls off too fast to affect it. Meanwhile $\mathcal{O}_{i_{1} \cdots i_{s}}^{(\beta)}$ undergoes an induced boundary gauge transformation $\delta \mathcal{O}_{i_{1} \cdots i_{s}}^{(\beta)}=\partial_{\left(i_{1}\right.} B_{\left.i_{2} \cdots i_{s}\right)}$ because the $B$-mode has the same fall-off as it.
} 
According to [12], $\frac{q^{\Delta}+q^{\bar{\Delta}}}{(1-q)^{d}}$ is exactly the Harish-Chandra character $\chi_{\Delta}^{\mathrm{HC}}(q) \equiv \operatorname{tr} q^{D},{ }^{10}$ for the scalar principal series, i.e. $\Delta \in \frac{d}{2}+i \mathbb{R}$ and the scalar complementary series, i.e. $0<$ $\Delta<d$. Note that the principal/complementary series condition just ensures a positive mass term for $\phi$, which in bulk is nothing but the unitarity condition. Therefore, the quasinormal character of a unitary scalar field is same as its Harish-Chandra character. For a massive spin- $s$ field, the story is almost the same except the $\alpha$-modes and $\beta$-modes have spin degeneracy $D_{s}^{d}$, the dimension of spin-s representation of $\mathrm{SO}(d)$. Taking into account this spin degeneracy, we obtain the quasinormal character of a spin- $s$ field of scaling dimension $\Delta$,

$$
\chi_{[\Delta, s]}^{\mathrm{QN}}(q)=D_{s}^{d} \frac{q^{\Delta}+q^{\bar{\Delta}}}{(1-q)^{d}}
$$

which is exactly the Harish-Chandra character for the spin- $s$ principal series, i.e. $\Delta \in \frac{d}{2}+i \mathbb{R}$ and the spin- $s$ complementary series, i.e. $1<\Delta<d-1$.

In the remaining part of this section, we will compute quasinormal characters for massless higher spin fields. In this case, the $\alpha$-part is easy because $\alpha_{i_{1} \cdots i_{s}}^{(s)}$ is a conserved current in the sense of (3.14). So for $i \omega_{n}^{\alpha}=d+s-2+n$ in the $\alpha$-tower, the degeneracy is $d_{n}^{\alpha}=\left(\begin{array}{c}n+d-1 \\ d-1\end{array}\right) D_{s}^{d}-\left(\begin{array}{c}n+d-2 \\ d-1\end{array}\right) D_{s-1}^{d}$, which yields

$$
\chi_{s}^{\mathrm{QN}, \alpha}(q)=\sum_{n \geq 0} d_{n}^{\alpha} q^{i \omega_{n}^{\alpha}}=\frac{D_{s}^{d} q^{d-2+s}-D_{s-1}^{d} q^{d-1+s}}{(1-q)^{d}}
$$

$\chi_{s}^{\mathrm{QN}, \alpha}(q)$ is the same as the $\mathrm{SO}(2, d)$ character corresponding to a massless spin- $s$ field in $\operatorname{AdS}_{d+1}[39,40]$. However, this is far from the corresponding $\mathrm{SO}(1, d+1)$ character $[12,14]$. In the notation of [14], the massless spin- $s$ representation of $\mathrm{SO}(1, d+1)$ is denoted by $D_{(\alpha ; p)}^{j}$, with $p=0, j=\frac{d-4}{2}$ for even $d, j=\frac{d-3}{2}$ for odd $d$ and $\alpha=(s, s, 0, \cdots, 0) .{ }^{11}$ In the notation of [12], this representation falls in the exceptional series with $\Delta=p=2$ and $\mathbb{Y}_{p}=\mathbb{Y}_{s s}$. The corresponding Harish-Chandra $\mathrm{SO}(1, d+1)$ character is

$$
\chi_{s}^{\mathrm{HC}}(q)=\left(1-(-1)^{d}\right) \frac{D_{s}^{d} q^{s+d-2}-D_{s-1}^{d} q^{s+d-1}}{(1-q)^{d}}+\sum_{n=2}^{d-2}(-)^{n} \frac{\mathcal{D}_{n} q^{n}}{(1-q)^{d}}
$$

where

$$
\mathcal{D}_{n}=\frac{\Gamma(d-3) s(s+1)(d+s-4)(d+s-3) D_{s s}^{d}}{\Gamma(n-1) \Gamma(\bar{n}-1)(s+n-2)(s+\bar{n}-2)(s+n-1)(s+\bar{n}-1)}, \quad \bar{n} \equiv d-n
$$

For $2 \leq n \leq\left\lfloor\frac{d}{2}\right\rfloor, \mathcal{D}_{n}$ is the dimension of $\mathrm{SO}(d)$ representation $\mathbb{Y}_{\left(s s, 1^{n-2}\right)}$, obtained by adding $n-2$ single-box rows to $\mathbb{Y}_{s s}$. (When $d=2 r$ and $n=r, \mathcal{D}_{r}$ is actually the dimension of $\left.\mathbb{Y}_{(s s 1 \cdots 1,+1)} \oplus \mathbb{Y}_{(s s 1 \cdots 1,-1)}\right)$. These values can be easily extended to $\left\lfloor\frac{d}{2}\right\rfloor+1 \leq n \leq d-2$ by the manifest $n \leftrightarrow d-n$ symmetry of $\mathcal{D}_{n}$.

To compare the quasinormal character $\chi_{s}^{\mathrm{QN}}$ with the Harish-Chandra character $\chi_{s}^{\mathrm{HC}}$, we still need to figure out quasinormal spectrum of the $\gamma$-tower.

\footnotetext{
${ }^{10}$ In our convention, $D$ is an anti-hermitian operator in unitary representations.

${ }^{11}$ Here we use a different convention for the highest weight vector $\alpha$ compared to [14].
} 
Maxwell field. Let's start from a Maxwell field. At level 0, i.e. $i \omega=2$, the degeneracy is $d_{0}^{\gamma}=\left(\begin{array}{l}d \\ 2\end{array}\right)$ because $\gamma_{i j}^{(1)}$ carries the 2 -form representation of $\mathrm{SO}(d)$. At level 1 , generic descendants are of the form $P_{k} \gamma_{i j}^{(1)}$, corresponding to the $\mathrm{SO}(d)$ representation $\square \otimes \boxminus$. The 3 -form representation in this tensor product is vanishing due to Bianchi identity $P_{[k} \gamma_{i j]}^{(1)}=0$. Therefore the degeneracy of quasinormal modes with frequency $i \omega=3$ is

$$
d_{1}^{\gamma}=d\left(\begin{array}{l}
d \\
2
\end{array}\right)-\left(\begin{array}{l}
d \\
3
\end{array}\right)=2\left(\begin{array}{c}
d+1 \\
3
\end{array}\right)
$$

The descendants at level 2 are $P_{k} P_{\ell} \gamma_{i j}^{(1)}$, corresponding to the $\mathrm{SO}(d)$ representation $(\bullet \oplus \square) \otimes \boxminus$. Due to Bianchi identity, we would exclude terms like $P_{\ell} P_{[k} \gamma_{i j]}^{(1)}$, that carries the $\square \otimes \exists$ representation. However, this is overcounting because the 4-form representation in this tensor product, carried by $P_{[\ell} P_{k} \gamma_{i j]}^{(1)}$, vanishes automatically without using Bianchi identity. Therefore the degeneracy of quasinormal modes with frequency $i \omega=4$ is

$$
d_{2}^{\gamma}=\left(\begin{array}{l}
d+1 \\
d-1
\end{array}\right)\left(\begin{array}{l}
d \\
2
\end{array}\right)-d\left(\begin{array}{l}
d \\
3
\end{array}\right)+\left(\begin{array}{l}
d \\
4
\end{array}\right)=3\left(\begin{array}{c}
d+2 \\
4
\end{array}\right)
$$

At any level $n$, using the same argument, we obtain the degeneracy of quasinormal modes with frequency $i \omega=2+n$

$$
d_{n}^{\gamma}=\sum_{k=0}^{n}(-)^{k}\left(\begin{array}{c}
d \\
k+2
\end{array}\right)\left(\begin{array}{c}
n+d-1-k \\
d-1
\end{array}\right)=(n+1)\left(\begin{array}{l}
n+d \\
d-2
\end{array}\right)
$$

which leads to the $\gamma$-tower quasinormal character

$$
\chi_{1}^{\mathrm{QN}, \gamma}(q)=\sum_{n \geq 0}(n+1)\left(\begin{array}{l}
n+d \\
d-2
\end{array}\right) q^{n+2}=1-\frac{1-d q}{(1-q)^{d}}
$$

Combining eq. (5.5) for $s=1$ and eq. (5.11), we get the full quasinormal character of a Maxwell field

$$
\chi_{1}^{\mathrm{QN}}(q)=\chi_{1}^{\mathrm{QN}, \alpha}(q)+\chi_{1}^{\mathrm{QN}, \gamma}(q)=1-\frac{1-d q}{(1-q)^{d}}+\frac{d q^{d-1}-q^{d}}{(1-q)^{d}}
$$

On the other hand, since $\mathcal{D}_{n}$ reduces to $\left(\begin{array}{l}d \\ n\end{array}\right)$ when $s=1$, the Harish-Chandra character (5.6) for $s=1$ is

$$
\chi_{1}^{\mathrm{HC}}(q)=\frac{d q^{d-1}-q^{d}}{(1-q)^{d}}+\sum_{n=2}^{d}(-)^{n} \frac{\left(\begin{array}{l}
d \\
n
\end{array}\right) q^{n}}{(1-q)^{d}}=\chi_{1}^{\mathrm{QN}}(q)
$$

Again, quasinormal character $=$ Harish-Chandra character. In appendix $\mathrm{B}$, we will show that $\chi_{1}^{\mathrm{QN}}(q)$ is also consistent with the spin-1 quasinormal spectrum in [7]. 
Higher spin fields. To check $\chi_{s}^{\mathrm{QN}}=\chi_{s}^{\mathrm{HC}}$ for any $s \geq 2$, it is easier to use a different but equivalent expression of $\chi_{s}^{\mathrm{HC}}$ found in [16]:

$$
\chi_{s}^{\mathrm{HC}}(q)=\frac{D_{s}^{d} q^{d+s-2}-D_{s-1}^{d} q^{s+d-1}}{(1-q)^{d}}+\left[\frac{D_{s}^{d} q^{2-s}-D_{s-1}^{d} q^{1-s}}{(1-q)^{d}}\right]_{+}
$$

where [ ]+ is a linear operator that sends $q^{k} \rightarrow-q^{-k}$ for $k<0$ and drops the constant term while acting on a Laurent series around $q=0$. As a very simple example, $\left[q^{-1}+1+q\right]_{+}=0$. Notice that the first term of $\chi_{s}^{\mathrm{HC}}(q)$ is the same as $\chi_{s}^{\mathrm{QN}, \alpha}(q)$, so it suffices to compare the second term with $\chi_{s}^{\mathrm{QN}, \gamma}(q)$. Expand the second term into a Taylor series around $q=0$ :

$$
\left[\frac{D_{s}^{d} q^{2-s}-D_{s-1}^{d} q^{1-s}}{(1-q)^{d}}\right]_{+} \equiv \sum_{n \geq 0} b_{n} q^{2+n}
$$

With some simple algebra, one can show that $b_{0}=D_{s s}^{d}, b_{1}=D_{s+1, s}^{d}+D_{s, s-1}^{d}$ and furthermore $b_{n}$ satisfies the following recurrence relation

$$
b_{n}-b_{n-2}=D_{s}^{d}\left(D_{s+n}^{d}+D_{s-n-2}^{d}\right)-D_{s-1}^{d}\left(D_{s+n+1}^{d}+D_{s-n-1}^{d}\right)
$$

Using the tensor product decomposition of $\mathbb{Y}_{s} \otimes \mathbb{Y}_{t}$ (assuming $t \leq s$ )

$$
\mathbb{Y}_{s} \otimes \mathbb{Y}_{t}=\bigoplus_{\ell=0}^{t} \bigoplus_{m=0}^{t-\ell} \mathbb{Y}_{s+t-2 \ell-m, m}
$$

the products of dimensions in eq. (5.16) can be rewritten as a summation

$$
b_{n}-b_{n-2}=\sum_{\ell=0}^{s} D_{s-\ell+n, s-\ell}^{d}-\sum_{\ell=0}^{s-n-1} D_{s-\ell-1, s-n-1-\ell}^{d}=\left(\sum_{\ell=0}^{s}-\sum_{\ell=n+1}^{s}\right) D_{s-\ell+n, s-\ell}^{d}
$$

When $n \geq s$ the second sum in the bracket vanishes and when $n \leq s$, the first sum over $\ell$ gets truncated at $\ell=n$. Altogether,

$$
b_{n}-b_{n-2}=\sum_{\ell=0}^{\min (n, s)} D_{s-\ell+n, s-\ell}^{d}
$$

On the quasinormal modes side, since the primary $\gamma^{(s)}$ carries $\mathbb{Y}_{s s}$ representation, the degeneracy at level 0 is $d_{0}^{\gamma}=D_{s s}^{d}=b_{0}$. At level 1 , the descendants $P_{k} \gamma_{i_{1} j_{1}, \cdots, i_{s} j_{s}}^{(s)}$ are represented by $\mathbb{Y}_{1} \otimes \mathbb{Y}_{s s}=\mathbb{Y}_{s+1, s} \oplus \mathbb{Y}_{s, s-1} \oplus \mathbb{Y}_{s, s, 1}$. Due to Bianchi identity, the three-row summand in this tensor product vanishes and the level 1 descendants only carry the $\mathbb{Y}_{s+1, s} \oplus$ $\mathbb{Y}_{s, s-1}$ representation. So the degeneracy of quasinormal frequency $i \omega=3$ is $d_{1}^{\gamma}=D_{s+1, s}^{d}+$ $D_{s, s-1}^{d}=b_{1}$. At higher levels, we aim to derive a recurrence relation for the degeneracy $d_{n}^{\gamma}$. For example, at level $n$, the descendants are of the form $P_{\ell_{1}} \cdots P_{\ell_{n}} \gamma_{i_{1} j_{1}, \cdots, i_{s} j_{s}}^{(s)}$, where $P_{\ell_{1}} \cdots P_{\ell_{n}}$ should be understood group theoretically as the symmetrized tensor product of $n$ spin-1 representations. Compared to level $(n-2)$, the additional representation structure is $\mathbb{Y}_{n} \otimes \mathbb{Y}_{s s}$ where $\mathbb{Y}_{n}$ corresponds to the traceless part of $P_{\ell_{1}} \cdots P_{\ell_{n}}$ and $\mathbb{Y}_{s s}$ corresponds to $\gamma^{(s)}$. Due to Bianchi identity, only two-row representations in the tensor 


\begin{tabular}{|c|c|c|c|}
\hline Primaries & $i \omega_{\mathrm{QN}}$ & $\mathrm{SO}(d)$ representation & constraint \\
\hline$\alpha_{i_{1} \cdots i_{s}}^{\mu_{1} \cdots \mu_{s}}$ & $d+s-2$ & $\mathbb{Y}_{s}$ & conservation law \\
\hline$\gamma_{i_{1} \cdots j_{1}, \cdots, i_{s} j_{s}}^{\mu_{1}}$ & 2 & $\mathbb{Y}_{s s}$ & Bianchi identity \\
\hline
\end{tabular}

Table 1: A brief summary about the physical primary quasinormal modes of a massless spin- $s$ gauge field in $\mathrm{dS}_{d+1}(d \geq 4) . \mu_{k}$ are bulk spin indices and $i_{k}, j_{k}$ indicate the $\mathrm{SO}(d)$ representation whose dimension gives the degeneracy.

product decomposition of $\mathbb{Y}_{n} \otimes \mathbb{Y}_{s s}$ are nonvanishing. These two-row representations are exactly $\oplus_{\ell=0}^{\min (n, s)} \mathbb{Y}_{s-\ell+n, s-\ell}$, which yields $d_{n}^{\gamma}-d_{n-2}^{\gamma}=b_{n}-b_{n-2}$ and furthermore $b_{n}=d_{n}^{\gamma}$ for $n \geq 0$. Altogether, we can conclude

$$
\chi_{s}^{\mathrm{QN}}(q)=\chi_{s}^{\mathrm{HC}}(q)
$$

\section{Conclusion and outlook}

In this paper, we present an algebraic method of constructing quasinormal modes of massless higher spin fields in the southern static patch of $\mathrm{dS}_{d+1}$ using ambient space formalism. With the action of isometry group $\mathrm{SO}(1, d+1)$, the whole quasinormal spectrum can be built from two primary quasinormal modes, whose properties are summarized in the table. 1 (assuming $d \geq 4$ )

For example, when $s=2$, the primary $\alpha$-modes $\alpha_{i_{1} i_{2}}^{\mu \nu}$ have quasinormal frequency $\omega_{Q N}=-i d$ and degeneracy $D_{2}^{d}=\frac{(d+2)(d-1)}{2}$ because the $i_{1}, i_{2}$ indices transform as a spin-2 representation of $\mathrm{SO}(d)$. The conservation law means that $P_{i_{1}} \alpha_{i_{1} i_{2}}^{\mu \nu}$ is pure gauge and hence should be excluded from the physical spectrum of quasinormal modes. On the other hand, the primary $\gamma$-modes $\gamma_{i_{1} j_{1}, i_{2} j_{2}}^{\mu \nu}$ have quasinormal frequency $\omega_{Q N}=-2 i$ and degeneracy $D_{22}^{d}=\frac{1}{12}(d+2)(d+1) d(d-3)$ because the indices $\left[i_{1}, j_{1}\right],\left[i_{2}, j_{2}\right]$ transform as Weyl tensor under $\mathrm{SO}(d)$. This also explains the Bianchi identity $P_{[k} \gamma_{\left.i_{1} j_{1}\right], i_{2} j_{2}}^{\mu \nu}=0$.

With the higher spin quasinormal modes known, we define a quasinormal character $\chi_{s}^{\mathrm{QN}}(q)$, cf. (5.1) that encodes precisely the information of quasinormal spectrum. We show that $\chi_{s}^{\mathrm{QN}}(q)$ is equal to the Harish-Chandra group character $\chi_{s}^{\mathrm{HC}}(q)$ of the unitary massless spin- $s \mathrm{SO}(1, d+1)$ representation. In other words, the pure group theoretical object $\chi_{s}^{\mathrm{HC}}(q)$ knows everything about the physical quasinormal spectrum.

Our algebraic approach to quasinormal modes has some potential generalizations and applications which will be left to investigate in the future:

- Construct quasinormal modes of fields carrying other unitary representations, for example partially massless fields or discrete series fields. The generalization to partially massless fields should be more or less straightforward. In particular, the construction of the primary $\alpha$-modes would be the same except the conservation law being replaced by a multiply-conservation equation [41]:

$$
P_{i_{1}} \cdots P_{i_{s-t}} \alpha_{i_{1} \cdots i_{s}}^{\mu_{1} \cdots \mu_{s}}=\text { pure gauge }
$$


where $t$ is the depth. For the primary $\gamma$-modes, the higher spin Weyl tensor used in eq. (3.34) is expected to be replaced by its partially massless counterpart which carries $\mathbb{Y}_{s, t+1}$ representation of $\mathrm{SO}(d)[24,25,42]$. The discrete series case should be different because it is labelled by a maximal height Young diagram. As a result, neither of the primary quasinormal modes can be a curvature like object. This is also confirmed from the character side [12].

- Generalize the "quasinormal quantization" $[9,10]$ to massless higher spin gauge fields in any higher dimension. It is well known that quasinormal modes are nonnormalizable with respect to the standard Klein-Gordon inner product. However, it is noticed in $[9,10]$ that, at least for light scalar fields in $\mathrm{dS}_{4}$, there is the so-called "R-norm" such that the quasinormal modes become normalizable and $\mathrm{SO}(1,4)$ is effectively Wick rotated to $\mathrm{SO}(2,3)$. Granting the existence of "R-norm" in higher dimensions for massless higher spin fields that maps $\mathrm{SO}(1, d+1)$ to $\mathrm{SO}(2, d)$, then the $\gamma$-tower of quasinormal modes carries the $\left[\Delta=2, \mathbb{Y}_{s s}\right]$ representation of $\mathrm{SO}(2, d)$, that is below the unitarity bound for sufficiently large $s$. This simple argument seems to question the naive generalization of "R-norm".

- In this paper, we have focused on Harish-Chandra character $\chi_{R}(q)=\operatorname{tr}_{R} q^{D}$ with only the scaling operator $D$ turned on, where $R$ denotes some unitary irreducible representation. In general, we can also include $\mathrm{SO}(d)$ generators in the definition of characters:

$$
\chi_{R}^{\mathrm{HC}}(q, x)=\operatorname{tr}_{R}\left(q^{D} x_{1}^{J_{1}} \cdots x_{r}^{J_{r}}\right), \quad r=\left\lfloor\frac{d}{2}\right\rfloor
$$

where $J_{i}=L_{2 i-1,2 i}$ span the Cartan algebra of $\mathrm{SO}(d)$ and $x_{i}$ are auxiliary variables. For example, for spin- $s$ principal series or complementary series, the full character $(6.2)$ reads $[12,14]$

$$
\chi_{[\Delta, s]}^{\mathrm{HC}}(q, x)=\left(q^{\Delta}+q^{\bar{\Delta}}\right) \chi_{\mathbb{Y}_{s}}^{\mathrm{SO}(d)}(x) P_{d}(q, x)
$$

where $\chi_{\mathbb{Y}}^{\mathrm{SO}(d)}(x) \equiv \operatorname{tr}_{\mathbb{Y}} x_{1}^{J_{1}} \cdots x_{r}^{J_{r}}$ denotes the $\mathrm{SO}(d)$ character of spin- $\mathbb{Y}$ representation and

$$
P_{d}(q, x)=\frac{1}{\prod_{i=1}^{r}\left(1-x_{i} q\right)\left(1-x_{i}^{-1} q\right)} \times\left\{\begin{aligned}
1, & & d=2 r \\
\frac{1}{1-q}, & & d=2 r+1
\end{aligned}\right.
$$

For massless spin- $s$ representation, the full character originally computed in [14] is:

$$
\begin{aligned}
d=2 r+1: \quad \chi_{s}^{\mathrm{HC}}(q, x)= & 2\left(\chi_{\mathbb{Y}_{s}}^{\mathrm{SO}(d)}(x) q^{s+d-2}-\chi_{\mathbb{Y}_{s-1}}^{\mathrm{SO}(d)}(x) q^{s+d-1}\right) P_{d}(q, x) \\
& +\sum_{n=2}^{r}(-)^{n}\left(q^{n}-q^{d-n}\right) \chi_{\mathbb{Y}_{\left(s s, 1^{n-2}\right)}}^{\mathrm{SO}(d)}(x) P_{d}(q, x)
\end{aligned}
$$

and

$$
\begin{aligned}
d=2 r: \quad \chi_{s}^{\mathrm{HC}}(q, x)= & \sum_{n=2}^{r-1}(-)^{n}\left(q^{n}+q^{d-n}\right) \chi_{\mathbb{Y}_{\left(s s, 1^{n-2}\right)}}^{\mathrm{SO}(d)}(x) P_{d}(q, x) \\
& +(-)^{r} q^{r}\left(\chi_{\mathbb{Y}_{(s s 1, \cdots,+1)}}^{\mathrm{SO}(d)}(x)+\chi_{\mathbb{Y}_{(s s 1, \cdots,-1)}}^{\mathrm{SO}(d)}(x)\right) P_{d}(q, x)
\end{aligned}
$$


We conjecture that the full Harish-Chandra character encodes spin content of quasinormal modes. More precisely, expand $\chi_{s}^{\mathrm{HC}}(q, x)$ in terms of $q$ and $x_{i}$

$$
\chi_{s}^{\mathrm{HC}}(q, x)=\sum_{\omega, \mathbf{j}} d_{\omega, \mathbf{j}} q^{i \omega} x_{1}^{j_{1}} \cdots x_{r}^{j_{r}}, \quad \mathbf{j}=\left(j_{1}, \cdots, j_{r}\right)
$$

then $d_{\omega, \mathbf{j}}$ is conjectured to be the degeneracy of quasinormal modes with frequency $\omega$ and spin content $\mathbf{j}$.

\section{Acknowledgments}

I am grateful to Frederik Denef, Austin Joyce and Albert Law for numerous stimulating discussions, at different stages of this research project. I also thank Dionysios Anninos and Frederik Denef for reading the paper and providing precious comments. ZS was supported in part by the U.S. Department of Energy grant de-sc0011941.

\section{A From ambient space to intrinsic coordinate: Maxwell field}

In this appendix, we show the agreement between our algebraically constructed primary quasnormal modes and their intrinsic coordinate counterparts in literature for free Maxwell fields. According to [7], the quasinormal modes of Maxwell theory can be divided into the following two types:

$\mathrm{I}: \quad A_{t}^{(I)}=0, \quad A_{r}^{(I)}=R^{(I)}(r) Y^{\ell \sigma} e^{-i \omega t}, \quad A_{a}^{(I)}=\frac{r^{3-d}\left(1-r^{2}\right)}{\ell(\ell+d-2)} \partial_{r}\left(r^{d-1} R^{(I)}(r)\right) \partial_{\vartheta^{a}} Y^{\ell \sigma} e^{-i \omega t}$

where $\vartheta^{a}$ are the spherical coordinates on $S^{d-1}$ and $Y^{\ell \sigma}$ are scalar spherical harmonics with $\sigma$ being a collective symbol for the magnetic quantum numbers,

$$
\text { II : } \quad A_{t}^{(I I)}=A_{r}^{(I I)}=0, \quad A_{a}^{(I I)}=R^{(I I)}(r) Y_{a}^{\ell \sigma} e^{-i \omega t}
$$

where $Y_{a}^{\ell \sigma}$ are divergence-free vector spherical harmonics on $S^{d-1}$. In type I solutions, the radial function $R^{(I)}(r)$ is given by

$$
R^{(I)}(r)=r^{\ell-1}\left(1-r^{2}\right)^{\frac{i \omega}{2}} F\left(\frac{\ell+i \omega+d-2}{2}, \frac{\ell+i \omega+2}{2}, \frac{d}{2}+\ell, r^{2}\right)
$$

with the quasinormal frequency $\omega$ valued in

$$
i \omega_{\ell, n}^{I}=\ell+d-2+2 n, \quad i \tilde{\omega}_{\ell, n}^{I}=\ell+2+2 n
$$

In type II solutions, the radial function $R^{(I I)}(r)$ is given by

$$
R^{(I I)}(r)=r^{\ell+1}\left(1-r^{2}\right)^{\frac{i \omega}{2}} F\left(\frac{\ell+i \omega+d-1}{2}, \frac{\ell+i \omega+1}{2}, \frac{d}{2}+\ell, r^{2}\right)
$$

with the quasinormal frequency $\omega$ valued in

$$
i \omega_{\ell, n}^{I I}=\ell+d-1+2 n, \quad i \tilde{\omega}_{\ell, n}^{I I}=\ell+1+2 n
$$

In both type I and II, $\ell \geq 1$ and $n \geq 0$. In the following, we show that the primary quasinormal modes $\alpha_{i}^{(1)}$ agree with the type I solutions of frequency $i \omega_{1,0}^{I}$ and $\gamma_{i j}^{(1)}$ agrees with the type II solutions of frequency $i \tilde{\omega}_{1,0}^{I I}$. 
Match the primary $\boldsymbol{\alpha}_{i}^{(1)}$-mode. Using eq. (A.3), the type I quasinormal modes with $\ell=1$ and $i \omega=i \omega_{1,0}^{I}=d-1$ are

$$
A_{t}^{(I)}=0, \quad A_{r}^{(I)}=\frac{e^{-(d-1) t}}{\left(1-r^{2}\right)^{\frac{d-1}{2}}} Y^{1 \sigma}(\Omega), \quad A_{a}^{(I)}=\frac{r e^{-(d-1) t}}{\left(1-r^{2}\right)^{\frac{d-1}{2}}} \partial_{\vartheta^{a}} Y^{1 \sigma}(\Omega)
$$

On the other hand, the pull-back of $\alpha_{i}^{(1)}=\frac{X^{+} u_{i}-U^{+} x_{i}}{\left(X^{+}\right)^{d}} R^{d-2}$ yields

$$
\begin{aligned}
& \alpha_{i, t}^{(1)}=-\frac{x_{i} \partial_{t} X^{+}}{\left(X^{+}\right)^{d}}=-\frac{r \Omega_{i} e^{-(d-1) t}}{\left(1-r^{2}\right)^{\frac{d-1}{2}}} \\
& \alpha_{i, r}^{(1)}=\frac{X^{+} \partial_{r} x_{i}-x_{i} \partial_{r} X^{+}}{\left(X^{+}\right)^{d}}=\frac{\Omega_{i} e^{-(d-1) t}}{\left(1-r^{2}\right)^{\frac{d+1}{2}}} \\
& \alpha_{i, a}^{(1)}=\frac{X^{+} \partial_{\vartheta^{a}} x_{i}}{\left(X^{+}\right)^{d}}=\frac{r \partial_{\vartheta} \Omega_{i} e^{-(d-1) t}}{\left(1-r^{2}\right)^{\frac{d-1}{2}}}
\end{aligned}
$$

Naively, $A_{\mu}^{(I)}$ and $\alpha_{i, \mu}^{(1)}$ look different. This is because the former is solved in a modified Feynman gauge [43] while the latter follows from boundary-to-bulk propagator in de Donder gauge, which for spin-1 field is simply the Lorenz gauge. To compare the two results, we perform a gauge transformation $\alpha_{i, \mu}^{(1)} \rightarrow \tilde{\alpha}_{i, \mu}^{(1)}=\alpha_{i, \mu}^{(1)}+\partial_{\mu} \xi_{i}$ to set the $t$-component zero. The simplest choice of the gauge parameter is $\xi_{i}=\frac{1}{d-1} \alpha_{i, t}$. With this gauge choice, the new $\alpha_{i}^{(1)}$ modes become

$$
\tilde{\alpha}_{i, t}^{(1)}=0, \quad \tilde{\alpha}_{i, r}^{(1)}=\frac{d-2}{d-1} \frac{\Omega_{i} e^{-(d-1) t}}{\left(1-r^{2}\right)^{\frac{d-1}{2}}}, \quad \tilde{\alpha}_{i, a}^{(1)}=\frac{d-2}{d-1} \frac{r \partial_{\vartheta_{a}} \Omega_{i} e^{-(d-1) t}}{\left(1-r^{2}\right)^{\frac{d-1}{2}}}
$$

Since $\left\{\Omega_{i}\right\}_{i}$ and $\left\{Y^{1 \sigma}\right\}_{\sigma}$ are just different basis for the same vector space of spherical harmonics of eigenvalue $-(d-1)$ with respect to $\nabla_{S^{d-1}}^{2}$, eq. (A.7) and eq. (A.9) actually represent the same set of quasinormal modes.

Match the primary $\gamma_{i j}^{(1)}$-mode. Using eq. (A.5), the type II quasinormal modes with $\ell=1$ and $i \omega=i \tilde{\omega}_{1,0}^{I I}=2$ are

$$
A_{t}^{(I I)}=A_{r}^{(I I)}=0, \quad A_{a}^{(I I)}=\frac{r^{2} e^{-2 t}}{1-r^{2}} Y_{a}^{1 \sigma}(\Omega)
$$

On the other hand, the pull-back of $\gamma_{i j}^{(1)}=\frac{x_{i} u_{j}-x_{j} u_{i}}{\left(X^{+}\right)^{2}}$ yields

$$
\gamma_{i j, t}^{(1)}=\gamma_{i j, r}^{(1)}=0, \quad \gamma_{i j, a}^{(1)}=\frac{r^{2} e^{-2 t}}{1-r^{2}}\left(\Omega_{i} \partial_{\vartheta^{a}} \Omega_{j}-\Omega_{j} \partial_{\vartheta^{a}} \Omega_{i}\right)
$$

One can check directly that $\Sigma_{i j, a} \equiv \Omega_{i} \partial_{\vartheta^{a}} \Omega_{j}-\Omega_{j} \partial_{\vartheta^{a}} \Omega_{i}$ are indeed divergence-free vector harmonics of $\ell=1$. For example, let's consider the $d=3$ case where the vector harmonics are given by $Y_{a}^{\ell m}=\frac{1}{\sqrt{\ell(\ell+1)}} \epsilon_{a b} \nabla^{b} Y^{\ell m}[44]$ :

$$
Y_{a}^{1,0}=\frac{1}{2} \sqrt{\frac{3}{2 \pi}}\left(0, \sin ^{2} \theta\right), \quad Y_{a}^{1, \pm 1}=\frac{1}{4} \sqrt{\frac{3}{\pi}} e^{ \pm i \varphi}(-i, \pm \sin \theta \cos \theta)
$$


where $\vartheta^{a}=(\theta, \varphi)$ are the usual spherical coordinates on $S^{2}$. Meanwhile, by working out $\Sigma_{i j, a}$ explicitly, we obtain

$$
\Sigma_{12, a}=\left(0, \sin ^{2} \theta\right), \quad \Sigma_{23, a} \pm i \Sigma_{31, a}=\mp e^{ \pm i \varphi}(-i, \pm \sin \theta \cos \theta)
$$

Therefore, $\gamma_{i j, \mu}^{(1)}$ in (A.11) and $A_{\mu}^{(I I)}$ in (A.10) represent the same quasinormal modes.

\section{B Match quasinormal spectrums}

In the section 5, we defined a quasinormal character $\chi^{\mathrm{QN}}$ for a given quasinormal spectrum $\left\{\omega, d_{\omega}\right\}$, cf. (5.1). By definition, the correspondence between quasinormal characters and quasinormal spectrums is one-to-one. In this appendix, by using quasinormal characters, we show that our algebraic construction yields the same quasinormal spectrum as [7] for Maxwell fields and linearized gravity. On the algebraic side, the quasinormal character of a massless spin- $s$ field is shown to be given by eq. (5.14). In particular, for $s=1$ and $s=2$, the quasinormal characters read

$$
\chi_{1}^{\mathrm{QN}}(q)=\frac{d q^{d-1}-q^{d}}{(1-q)^{d}}+\frac{d q-1}{(1-q)^{d}}+1
$$

and

$$
\chi_{2}^{\mathrm{QN}}(q)=\frac{D_{2}^{d} q^{d}-D_{1}^{d} q^{d+1}}{(1-q)^{d}}+\frac{D_{2}^{d}-D_{1}^{d} q^{-1}}{(1-q)^{d}}+d\left(q+q^{-1}\right)+\frac{d^{2}-d+2}{2}
$$

where $D_{1}^{d}=d, \quad D_{2}^{d}=\frac{1}{2}(d+2)(d-1)$.

Maxwell fields. In the previous appendix, we have summarized the quasinormal modes of Maxwell fields computed in [7]. Here let's briefly recap the information about quasinormal frequencies:

$$
\begin{array}{lll}
\text { type I : } & i \omega_{\ell, n}^{I}=\ell+d-2+2 n, & i \tilde{\omega}_{\ell, n}^{I}=\ell+2+2 n \\
\text { type II : } & i \omega_{\ell, n}^{I I}=\ell+d-1+2 n, & i \tilde{\omega}_{\ell, n}^{I I}=\ell+1+2 n
\end{array}
$$

where $\ell \geq 1$ and $n \geq 0$. For fixed $\ell$ and $n$, each frequency of type I quasinormal modes has degeneracy $D_{\ell}^{d}$ because $\ell$ labels scalar spherical harmonics while each frequency of type II quasinormal modes has degeneracy $D_{\ell 1}^{d}$ because $\ell$ labels divergence-free vector spherical harmonics. So the quasinormal character associated to the spectrum (B.3) is

$$
\begin{aligned}
\chi_{1}^{\mathrm{QN}, \text { intrin }}(q) & \equiv \sum_{\ell=1}^{\infty} \sum_{n=0}^{\infty} D_{\ell}^{d}\left(q^{i \omega_{\ell, n}^{I}}+q^{i \tilde{\omega}_{\ell, n}^{I}}\right)+D_{\ell 1}^{d}\left(q^{i \omega_{\ell, n}^{I I}}+q^{i \tilde{\omega}_{\ell, n}^{I I}}\right) \\
& =\frac{q^{2}+q^{d-2}}{1-q^{2}} \sum_{\ell \geq 1} D_{\ell}^{d} q^{\ell}+\frac{q+q^{d-1}}{1-q^{2}} \sum_{\ell \geq 1} D_{\ell 1}^{d} q^{\ell}
\end{aligned}
$$

where the first sum over $\ell$ simply follows from

$$
\sum_{\ell \geq 0} D_{\ell}^{d} q^{\ell}=\frac{1+q}{(1-q)^{d-1}}
$$


The second sum over $\ell$ in (B.4) can be derived using

$$
D_{\ell s}^{d}=D_{\ell}^{d} D_{s}^{d-2}-D_{s-1}^{d} D_{\ell+1}^{d-2}
$$

In particular, when $s=1, D_{\ell 1}^{d}=(d-2) D_{\ell}^{d}-D_{\ell+1}^{d-2}$ and hence the second sum reduces to the (B.5) type:

$$
\sum_{\ell \geq 1} D_{\ell 1}^{d} q^{\ell}=(d-2) \frac{1+q}{(1-q)^{d-1}}-\left(\frac{1+q^{-1}}{(1-q)^{d-3}}-q^{-1}\right)
$$

Plugging (B.5) and (B.7) into the quasinormal character (B.4) yields

$$
\chi_{1}^{\mathrm{QN}, \text { intrin }}(q)=\frac{d q^{d-1}-q^{d}}{(1-q)^{d}}+\frac{d q-1}{(1-q)^{d}}+1=\chi_{1}^{\mathrm{QN}}(q)
$$

which shows the agreement between our algebraic method and the traditional analytical method on the quasinormal spectrum for Maxwell theory.

Linearized gravity. Quasinormal modes of linearized gravity are divided into three categories. The three types of fluctuation can be solved simultaneously by using the socalled Ishibashi-Kodama equation $[2,7,45]$. The quasinormal frequencies are:

Scalar type fluctuation : $\quad i \omega_{\ell, n}^{S}=\ell+d-2+2 n, \quad i \tilde{\omega}_{\ell, n}^{S}=\ell+2+2 n$

Vector type fluctuation : $\quad i \omega_{\ell, n}^{V}=\ell+d-1+2 n, \quad i \tilde{\omega}_{\ell, n}^{V}=\ell+1+2 n$

Tensor type fluctuation : $\quad i \omega_{\ell, n}^{T}=\ell+d+2 n, \quad i \tilde{\omega}_{\ell, n}^{T}=\ell+2 n$

where $\ell \geq 2$ and $n \geq 0$. In these 3 types of fluctuations, $\ell$ labels scalar spherical harmonics, divergence-free vector spherical harmonics and divergence-free tensor spherical harmonics on $S^{d-1}$ respectively and hence for fixed $\ell$ and $n$, each frequency has degeneracy $D_{\ell}^{d}, D_{\ell 1}^{d}$ and $D_{\ell 2}^{d}$ respectively. Altogether, the quasinormal character associated to the spectrum (B.9) is given by

$$
\begin{aligned}
\chi_{2}^{\mathrm{QN}, \text { intrin }}(q) & \equiv \sum_{\ell \geq 2, n \geq 0} D_{\ell}^{d}\left(q^{i \omega_{\ell n}^{S}}+q^{i \tilde{\omega}_{\ell n}^{S}}\right)+D_{\ell 1}^{d}\left(q^{i \omega_{\ell n}^{V}}+q^{i \tilde{\omega}_{\ell n}^{V}}\right)+D_{\ell 2}^{d}\left(q^{i \omega_{\ell n}^{T}}+q^{i \tilde{\omega}_{\ell n}^{T}}\right) \\
& =\frac{q^{2}+q^{d-2}}{1-q^{2}} \sum_{\ell \geq 2} D_{\ell}^{d} q^{\ell}+\frac{q+q^{d-1}}{1-q^{2}} \sum_{\ell \geq 2} D_{\ell 1}^{d} q^{\ell}+\frac{1+q^{d}}{1-q^{2}} \sum_{\ell \geq 2} D_{\ell 2}^{d} q^{\ell}
\end{aligned}
$$

where the first two series of $\ell$ are essentially computed in the Maxwell field case and the last series follows from eq. (B.6) with $s=2$ :

$$
\sum_{\ell \geq 2} D_{\ell 2}^{d} q^{\ell}=D_{2}^{d} \frac{1+q}{(1-q)^{d-1}}-d\left(\frac{1+q^{-1}}{(1-q)^{d-3}}-q^{-1}\right)+\frac{d(d-1)}{2}
$$

Combine the three series of $\ell$ in (B.10) and we obtain

$$
\chi_{2}^{\mathrm{QN}, \text { intrin }}(q)=\frac{D_{2}^{d}\left(q^{d}\right)-D_{1}^{d} q^{d+1}}{(1-q)^{d}}+\frac{D_{2}^{d}-D_{1}^{d} q^{-1}}{(1-q)^{d}}+d\left(q+q^{-1}\right)+\frac{d^{2}-d+2}{2}
$$


which is exactly $\chi_{2}^{\mathrm{QN}}(q)$. This computation confirms the match of quasinormal spectrum for linearized gravity. ${ }^{12}$

\section{Details of $\gamma_{i_{1} \ell_{1}, \cdots, i_{s} \ell_{s}}^{(s)}$}

The higher spin quasinormal mode $\gamma_{i_{1} \ell_{1}, \cdots, i_{s} \ell_{s}}^{(s)}$ defined by eq. (3.34) is rather schematic. In this appendix, we will write out its explicit form and then show various properties of it. Let's start from recollecting the definitions

$$
\begin{aligned}
\gamma_{i_{1} \ell_{1}, \cdots, i_{s} \ell_{s}}^{(s)}(X, U) & =\Pi_{s s} P_{i_{1}} \cdots P_{i_{s}}\left(-\log \left(X^{+}\right) \beta_{\ell_{1} \cdots \ell_{s}}^{(s)}\right)-\text { trace } \\
\beta_{\ell_{1} \cdots \ell_{s}}^{(s)}(X, U) & =\frac{1}{\left(X^{+}\right)^{2}}\left(X^{+} u_{\ell_{1}}-U^{+} x_{\ell_{1}}\right) \cdots\left(X^{+} u_{\ell_{s}}-U^{+} x_{\ell_{s}}\right)-\text { trace } \\
P_{i} & =-X^{-} \partial_{x^{i}}-2 x_{i} \partial_{X^{+}}-U^{-} \partial_{u^{i}}-2 u_{i} \partial_{U^{+}}
\end{aligned}
$$

where the projection operator $\Pi_{s s}$ antisymmetrizes $\left[i_{1}, \ell_{1}\right], \cdots,\left[i_{s}, \ell_{s}\right]$. Notice that $X^{-} \partial_{x^{i}}$ and $U^{-} \partial_{u^{i}}$ would introduce terms proportional $\delta_{i_{j} \ell_{k}}$ and $\delta_{i_{j} i_{k}}$. The former is killed by $\Pi_{s s}$ and the latter as a pure trace term also drops out in $\gamma_{i_{1} \ell_{1}, \cdots, i_{s} \ell_{s}}^{(s)}$. Therefore only $2 x_{i} \partial_{X^{+}}$and $2 u_{i} \partial_{U^{+}}$can have nonvanishing contributions to $\gamma_{i_{1} \ell_{1}, \cdots, i_{s} \ell_{s}}^{(s)}$ and $\gamma_{i_{1} \ell_{1}, \cdots, i_{s} \ell_{s}}^{(s)}$ is independent of $X^{-}$and $U^{-}$. As a result, $\gamma_{i_{1} \ell_{1}, \cdots, i_{s} \ell_{s}}^{(s)}$ has to be of the following form

$$
\begin{aligned}
\gamma_{i_{1} \ell_{1}, \cdots, i_{s} \ell_{s}}^{(s)}(X, U) & =\chi_{i_{1} \ell_{1}, \cdots, i_{s} \ell_{s}}^{(s)}(x, u) f\left(X^{+}, U^{+}\right) \\
\chi_{i_{1} \ell_{1}, \cdots, i_{s} \ell_{s}}^{(s)}(x, u) & \equiv\left(x_{i_{1}} u_{\ell_{1}}-u_{i_{1}} x_{\ell_{1}}\right) \cdots\left(x_{i_{s}} u_{\ell_{s}}-u_{i_{s}} x_{\ell_{s}}\right)-\text { trace }
\end{aligned}
$$

where $f\left(X^{+}, U^{+}\right)$is an unknown function to be fixed. With $x_{i}, u_{\ell}$ being $\mathrm{SO}(d)$ vectors, the tensor $\chi_{i_{1} \ell_{1}, \cdots, i_{s} \ell_{s}}^{(s)}(x, u)$ carries the $\mathbb{Y}_{s s}$ representation of $\mathrm{SO}(d)$. In particular, it satisfies the following set of equations which can be thought as the $\mathrm{SO}(d)$ analogue of uplift conditions and Pauli-Fierz conditions:

$$
\begin{aligned}
\partial_{x}^{2} \chi^{(s)}(x, u) & =\partial_{u}^{2} \chi^{(s)}(x, u)=\partial_{x} \cdot \partial_{u} \chi^{(s)}(x, u)=0 \\
x \cdot \partial_{u} \chi^{(s)}(x, u) & =u \cdot \partial_{x} \chi^{(s)}(x, u)=\left(x \cdot \partial_{x}-s\right) \chi^{(s)}(x, u)=\left(u \cdot \partial_{u}-s\right) \chi^{(s)}(x, u)=0
\end{aligned}
$$

where the subscripts of $\chi_{i_{1} \ell_{1}, \cdots, i_{s} \ell_{s}}^{(s)}$ are suppressed.

Using the definition (C.1), it is easy to check that $\gamma_{i_{1} \ell_{1}, \cdots, i_{s} \ell_{s}}^{(s)}$ satisfies the same conditions (2.6)-(2.10) as $\beta_{\ell_{1} \cdots \ell_{s}}^{(s)}$ by choosing $f$ properly. In particular, the homogeneity condition and the tangentiality condition yields

$$
X^{+} \partial_{U^{+}} f\left(X^{+}, U^{+}\right)=0, \quad X^{+} \partial_{X^{+}} f\left(X^{+}, U^{+}\right)=-2 f\left(X^{+}, U^{+}\right)
$$

\footnotetext{
${ }^{12}$ When $d=3$, the tensor type fluctuations doesn not exist because there is no divergence-free tensor harmonics on $S^{2}$. However, one can still recover the quasinormal character $\chi_{2}^{\mathrm{QN}}(q)$ for $d=3$ by counting quasinormal modes in the scalar and vector type fluctuations in this case.
} 
which have solution $f\left(X^{+}, U^{+}\right)=\frac{1}{\left(X^{+}\right)^{2}}$. Therefore, up to an unimportant normalization factor $c_{s}$, the explicit form of $\gamma_{i_{1} \ell_{1}, \cdots i_{s} \ell_{s}}^{(s)}$ is

$$
\gamma_{i_{1} \ell_{1}, \cdots, i_{s} \ell_{s}}^{(s)}=c_{s} \frac{\chi_{i_{1} \ell_{1}, \cdots, i_{s} \ell_{s}}^{(s)}(x, u)}{\left(X^{+}\right)^{2}}=c_{s} \frac{\left(x_{i_{1}} u_{\ell_{1}}-u_{i_{1}} x_{\ell_{1}}\right) \cdots\left(x_{i_{s}} u_{\ell_{s}}-u_{i_{s}} x_{\ell_{s}}\right)-\text { trace }}{\left(X^{+}\right)^{2}}
$$

For example, for a Maxwell filed, (C.8) is consistent with (3.19). More generally, all the $\gamma$-primaries that are generated by $\mathrm{SO}(d)$ action on (C.8) can be collectively expressed as

$$
\gamma^{(s)}(X, U)=\frac{T_{s s}(x, u)}{\left(X^{+}\right)^{2}}
$$

where $T_{s s}(x, u)$ is a polynomial in $x^{i}, u^{i}$ carrying the $\mathbb{Y}_{s s}$ representation of $\mathrm{SO}(d),{ }^{13}$ i.e. it satisfies

$$
T_{s s}(a x, b u)=(a b)^{s} T_{s s}(x, u), \quad u \cdot \partial_{x} T_{s s}(x, u)=\partial_{u}^{2} T_{s s}(x, u)=0
$$

All the linearly independent choices of $T_{s s}$ correspond to the degeneracy of $\gamma^{(s)}$.

Near horizon $\gamma_{i_{1} \ell_{1}, \cdots, i_{s} \ell_{s}}^{(s)}$ becomes singular because $X^{+} \rightarrow 0$. This singular behavior also exhibits the following in-going boundary condition:

$$
\gamma_{i_{1} \ell_{1}, \cdots, i_{s} \ell_{s}}^{(s)}(\rho \rightarrow \infty) \sim \frac{1}{\left(X^{+}\right)^{2}} \sim e^{-2(T-\rho)}
$$

where we can directly read off the quasinormal frequency $i \omega=2$.

The next task is to show that $\gamma_{i_{1} \ell_{1}, \cdots, i_{s} \ell_{s}}^{(s)}$ is primary up to gauge transformation by using its explicit form (C.8) (we can also use (C.9) with $T_{s s}(x, u)$ subject to (C.10)). Acting the special conformal transformation $K_{m}$ on $\gamma_{i_{1} \ell_{1}, \cdots, i_{s} \ell_{s}}^{(s)}$, we get (dropping the normalization constant $c_{s}$ )

$$
\begin{aligned}
K_{m} \gamma_{i_{1} \ell_{1}, \cdots, i_{s} \ell_{s}}^{(s)} & =\frac{1}{X^{+}} \partial_{x^{m}} \chi_{i_{1} \ell_{1}, \cdots, i_{s} \ell_{s}}^{(s)}+\frac{U^{+}}{\left(X^{+}\right)^{2}} \partial_{u^{m}} \chi_{i_{1} \ell_{1}, \cdots, i_{s} \ell_{s}}^{(s)} \\
& =U \cdot \partial_{X}\left(-\frac{\partial_{u^{m}} \chi_{i_{1} \ell_{1}, \cdots, i_{s} \ell_{s}}^{(s)}}{X^{+}}\right)+\frac{1}{X^{+}}\left(u \cdot \partial_{x} \partial_{u^{m}}+\partial_{x^{m}}\right) \chi_{i_{1} \ell_{1}, \cdots, i_{s} \ell_{s}}^{(s)}
\end{aligned}
$$

where we have replaced $U \cdot \partial_{X}$ by $u \cdot \partial_{x}$ in the second term of the second line because $\chi_{i_{1} \ell_{1}, \cdots i_{s} \ell_{s}}^{(s)}$ only depends on $x^{i}, u^{i}$. In addition, noticing that $u \cdot \partial_{x}$ kills $\chi_{i_{1} \ell_{1}, \cdots i_{s} \ell_{s}}^{(s)}$, the product of operators $u \cdot \partial_{x} \partial_{u^{m}}$ can be replaced by the corresponding commutator $\left[u \cdot \partial_{x}, \partial_{u^{m}}\right]=-\partial_{x^{m}}$ which cancels the other derivative with respect to $x^{m}$. Altogether, $\gamma_{i_{1} \ell_{1}, \cdots i_{s} \ell_{s}}^{(s)}$ is a primary quasinormal mode in the sense that $K_{m} \gamma_{i_{1} \ell_{1}, \cdots i_{s} \ell_{s}}^{(s)}$ can be removed by a gauge transformation

$$
K_{m} \gamma_{i_{1} \ell_{1}, \cdots, i_{s} \ell_{s}}^{(s)}(X, U)=U \cdot \partial_{X}\left(-c_{s} \frac{\partial_{u^{m}} \chi_{i_{1} \ell_{1}, \cdots, i_{s} \ell_{s}}^{(s)}(x, u)}{X^{+}}\right)
$$

where we have restored the normalization constant $c_{s}$.

\footnotetext{
${ }^{13}$ Taking eq. (C.9) as an ansatz of quasinormal modes (which satisfy the in-going boundary condition automatically as we will see below), then it is easy to show that uplift conditions and Pauli-Fierz conditions are equivalent to $T_{s s}(x, u)$ carrying the $\mathbb{Y}_{s s}$ representation of $\mathrm{SO}(d)$.
} 
Open Access. This article is distributed under the terms of the Creative Commons Attribution License (CC-BY 4.0), which permits any use, distribution and reproduction in any medium, provided the original author(s) and source are credited.

\section{References}

[1] LIGO SCIENTIFIC and VIRGO collaborations, Tests of general relativity with GW150914, Phys. Rev. Lett. 116 (2016) 221101 [Erratum ibid. 121 (2018) 129902] [arXiv:1602.03841] [INSPIRE].

[2] J. Natario and R. Schiappa, On the classification of asymptotic quasinormal frequencies for d-dimensional black holes and quantum gravity, Adv. Theor. Math. Phys. 8 (2004) 1001 [hep-th/0411267] [INSPIRE].

[3] E. Berti, V. Cardoso and A.O. Starinets, Quasinormal modes of black holes and black branes, Class. Quant. Grav. 26 (2009) 163001 [arXiv:0905.2975] [INSPIRE].

[4] K.D. Kokkotas and B.G. Schmidt, Quasinormal modes of stars and black holes, Living Rev. Rel. 2 (1999) 2 [gr-qc/9909058] [INSPIRE].

[5] R.A. Konoplya and A. Zhidenko, Quasinormal modes of black holes: From astrophysics to string theory, Rev. Mod. Phys. 83 (2011) 793 [arXiv:1102.4014] [InSPIRE].

[6] P.R. Brady, C.M. Chambers, W.G. Laarakkers and E. Poisson, Radiative falloff in Schwarzschild-de Sitter space-time, Phys. Rev. D 60 (1999) 064003 [gr-qc/9902010] [INSPIRE].

[7] A. Lopez-Ortega, Quasinormal modes of D-dimensional de Sitter spacetime, Gen. Rel. Grav. 38 (2006) 1565 [gr-qc/0605027] [INSPIRE].

[8] A. Lopez-Ortega, On the quasinormal modes of the de Sitter spacetime, Gen. Rel. Grav. 44 (2012) 2387 [arXiv: 1207.6791] [inSPIRE].

[9] G.S. Ng and A. Strominger, State/Operator Correspondence in Higher-Spin dS/CFT, Class. Quant. Grav. 30 (2013) 104002 [arXiv:1204.1057] [INSPIRE].

[10] D.L. Jafferis, A. Lupsasca, V. Lysov, G.S. Ng and A. Strominger, Quasinormal quantization in de Sitter spacetime, JHEP 01 (2015) 004 [arXiv:1305.5523] [INSPIRE].

[11] M.R. Tanhayi, Quasinormal modes in de Sitter space: Plane wave method, Phys. Rev. D 90 (2014) 064010 [arXiv: 1402 .2893] [INSPIRE].

[12] T. Basile, X. Bekaert and N. Boulanger, Mixed-symmetry fields in de Sitter space: a group theoretical glance, JHEP 05 (2017) 081 [arXiv: 1612.08166] [INSPIRE].

[13] V.K. Dobrev, G. Mack, V.B. Petkova, S.G. Petrova and I.T. Todorov, Harmonic Analysis on the n-Dimensional Lorentz Group and Its Application to Conformal Quantum Field Theory, vol. 63 (1977), [DOI] [INSPIRE].

[14] T. Hirai, The characters of irreducible representations of the Lorentz group of $n$-th order, Proc. Japan Acad. 41 (1965) no. 7, 526.

[15] T. Hirai, On irreducible representations of the Lorentz group of $n$-th order, Proc. Japan Acad. 38 (1962) no. 6, 258.

[16] D. Anninos, F. Denef, Y.T.A. Law and Z. Sun, Quantum de Sitter horizon entropy from quasicanonical bulk, edge, sphere and topological string partition functions, arXiv:2009.12464 [INSPIRE]. 
[17] E. Cotton, Sur les varietés à trois dimensions, Ann. Fac. d. Sc. Toulouse 2e série, 1 (1899) 385 .

[18] S.J. Aldersley, Comments on certain divergence-free tensor densities in a 3-space, J. Math. Phys. 20 (1979) 1905.

[19] T. Damour and S. Deser, Geometry of spin 3 gauge theories, Annales de l'I.H.P. Physique théorique 47 (1987) 277.

[20] C.N. Pope and P.K. Townsend, Conformal Higher Spin in $(2+1)$-dimensions, Phys. Lett. B 225 (1989) 245 [INSPIRE].

[21] M. Henneaux, S. Hörtner and A. Leonard, Higher Spin Conformal Geometry in Three Dimensions and Prepotentials for Higher Spin Gauge Fields, JHEP 01 (2016) 073 [arXiv: 1511.07389] [INSPIRE].

[22] R.R. Metsaev, Massless mixed symmetry bosonic free fields in d-dimensional anti-de Sitter space-time, Phys. Lett. B 354 (1995) 78 [InSPIRE].

[23] R.R. Metsaev, Arbitrary spin massless bosonic fields in d-dimensional anti-de Sitter space, Lect. Notes Phys. 524 (1999) 331 [hep-th/9810231] [INSPIRE].

[24] N. Boulanger, C. Iazeolla and P. Sundell, Unfolding Mixed-Symmetry Fields in AdS and the BMV Conjecture: I. General Formalism, JHEP 07 (2009) 013 [arXiv:0812.3615] [INSPIRE].

[25] N. Boulanger, C. Iazeolla and P. Sundell, Unfolding Mixed-Symmetry Fields in AdS and the BMV Conjecture. II. Oscillator Realization, JHEP 07 (2009) 014 [arXiv:0812.4438] [INSPIRE].

[26] K.B. Alkalaev and M. Grigoriev, Unified BRST description of AdS gauge fields, Nucl. Phys. B 835 (2010) 197 [arXiv:0910.2690] [INSPIRE].

[27] K. Alkalaev and M. Grigoriev, Unified BRST approach to (partially) massless and massive AdS fields of arbitrary symmetry type, Nucl. Phys. B 853 (2011) 663 [arXiv:1105.6111] [INSPIRE].

[28] C. Fronsdal, Singletons and Massless, Integral Spin Fields on de Sitter Space, Phys. Rev. D 20 (1979) 848 [INSPIRE].

[29] X. Bekaert and E. Meunier, Higher spin interactions with scalar matter on constant curvature spacetimes: conserved current and cubic coupling generating functions, JHEP 11 (2010) 116 [arXiv:1007.4384] [INSPIRE].

[30] C. Sleight, Metric-like Methods in Higher Spin Holography, PoS Modave2016 (2017) 003 [arXiv: 1701.08360] [INSPIRE].

[31] C. Sleight and M. Taronna, Feynman rules for higher-spin gauge fields on $A d S_{d+1}$, JHEP 01 (2018) 060 [arXiv: 1708.08668] [inSPIRE].

[32] A. Mikhailov, Notes on higher spin symmetries, hep-th/0201019 [INSPIRE].

[33] M.S. Costa, V. Gonçalves and J. Penedones, Spinning AdS Propagators, JHEP 09 (2014) 064 [arXiv: 1404.5625] [inSPIRE].

[34] V.K. Dobrev, V.B. Petkova, S.G. Petrova and I.T. Todorov, Dynamical Derivation of Vacuum Operator Product Expansion in Euclidean Conformal Quantum Field Theory, Phys. Rev. D 13 (1976) 887 [inSPIRE]. 
[35] X. Bekaert and N. Boulanger, Tensor gauge fields in arbitrary representations of $G L(D, R)$ : Duality and Poincaré lemma, Commun. Math. Phys. 245 (2004) 27 [hep-th/0208058] [INSPIRE].

[36] V.E. Didenko and E.D. Skvortsov, Elements of Vasiliev theory, arXiv:1401.2975 [INSPIRE].

[37] R. Bousso, A. Maloney and A. Strominger, Conformal vacua and entropy in de Sitter space, Phys. Rev. D 65 (2002) 104039 [hep-th/0112218] [INSPIRE].

[38] D. Anninos, F. Denef, R. Monten and Z. Sun, Higher Spin de Sitter Hilbert Space, JHEP 10 (2019) 071 [arXiv: 1711.10037] [INSPIRE].

[39] F.A. Dolan, Character formulae and partition functions in higher dimensional conformal field theory, J. Math. Phys. 47 (2006) 062303 [hep-th/0508031] [INSPIRE].

[40] G.W. Gibbons, M.J. Perry and C.N. Pope, Partition functions, the Bekenstein bound and temperature inversion in anti-de Sitter space and its conformal boundary, Phys. Rev. D $\mathbf{7 4}$ (2006) 084009 [hep-th/0606186] [INSPIRE].

[41] L. Dolan, C.R. Nappi and E. Witten, Conformal operators for partially massless states, JHEP 10 (2001) 016 [hep-th/0109096] [INSPIRE].

[42] K. Hinterbichler and A. Joyce, Manifest Duality for Partially Massless Higher Spins, JHEP 09 (2016) 141 [arXiv: 1608.04385] [INSPIRE].

[43] L.C.B. Crispino, A. Higuchi and G.E.A. Matsas, Quantization of the electromagnetic field outside static black holes and its application to low-energy phenomena, Phys. Rev. D 63 (2001) 124008 [Erratum ibid. 80 (2009) 029906] [gr-qc/0011070] [INSPIRE].

[44] A. Higuchi, Symmetric Tensor Spherical Harmonics on the $N$ Sphere and Their Application to the de Sitter Group SO(N,1), J. Math. Phys. 28 (1987) 1553 [Erratum ibid. 43 (2002) 6385] [INSPIRE].

[45] H. Kodama and A. Ishibashi, Master equations for perturbations of generalized static black holes with charge in higher dimensions, Prog. Theor. Phys. 111 (2004) 29 [hep-th/0308128] [INSPIRE]. 